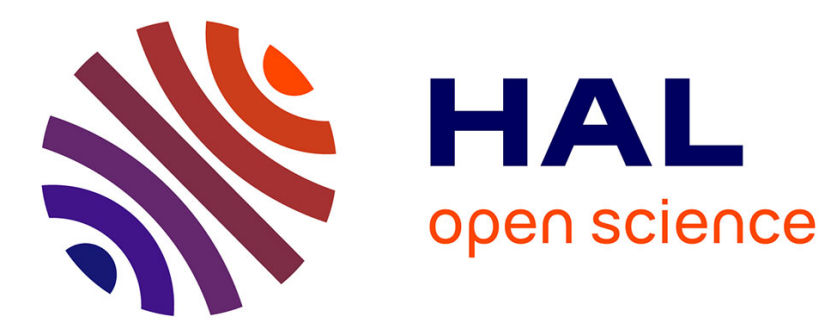

\title{
Sensitivity of aerodynamic forces in laminar and turbulent flow past a square cylinder
}

Philippe Meliga, Edouard Boujo, Gregory Pujals, François Gallaire

\section{To cite this version:}

Philippe Meliga, Edouard Boujo, Gregory Pujals, François Gallaire. Sensitivity of aerodynamic forces in laminar and turbulent flow past a square cylinder. Physics of Fluids, 2014, 26, pp.26,104101. 10.1063/1.4896941. hal-01082600v2

\section{HAL Id: hal-01082600 \\ https://hal.science/hal-01082600v2}

Submitted on 15 Nov 2014

HAL is a multi-disciplinary open access archive for the deposit and dissemination of scientific research documents, whether they are published or not. The documents may come from teaching and research institutions in France or abroad, or from public or private research centers.
L'archive ouverte pluridisciplinaire HAL, est destinée au dépôt et à la diffusion de documents scientifiques de niveau recherche, publiés ou non, émanant des établissements d'enseignement et de recherche français ou étrangers, des laboratoires publics ou privés. 


\title{
Sensitivity of aerodynamic forces in laminar and turbulent flow past a square cylinder
}

\author{
Philippe Meliga, ${ }^{1}$ Edouard Boujo, ${ }^{2}$ Gregory Pujals, ${ }^{3}$ and François Gallaire ${ }^{2}$ \\ ${ }^{1}$ Aix Marseille Université, CNRS, Centrale Marseille, M2P2 UMR 7340, \\ 13451 Marseille, France \\ ${ }^{2}$ LFMI École Polytechnique Fédérale de Lausanne, CH1015 Lausanne, Switzerland \\ ${ }^{3}$ PSA Peugeot Citroën, Centre Technique de Velizy, 78943 Vélizy-Villacoublay Cedex, France
}

(Received 13 February 2014; accepted 11 September 2014;

published online 14 October 2014)

\begin{abstract}
We use adjoint-based gradients to analyze the sensitivity of the drag force on a square cylinder. At $R e=40$, the flow settles down to a steady state. The quantity of interest in the adjoint formulation is the steady asymptotic value of drag reached after the initial transient, whose sensitivity is computed solving a steady adjoint problem from knowledge of the stable base solution. At $R e=100$, the flow develops to the timeperiodic, vortex-shedding state. The quantity of interest is rather the time-averaged mean drag, whose sensitivity is computed integrating backwards in time an unsteady adjoint problem from knowledge of the entire history of the vortex-shedding solution. Such theoretical frameworks allow us to identify the sensitive regions without computing the actually controlled states, and provide a relevant and systematic guideline on where in the flow to insert a secondary control cylinder in the attempt to reduce drag, as established from comparisons with dedicated numerical simulations of the two-cylinder system. For the unsteady case at $R e=100$, we also compute an approximation to the mean drag sensitivity solving a steady adjoint problem from knowledge of only the mean flow solution, and show the approach to carry valuable information in view of guiding relevant control strategy, besides reducing tremendously the related numerical effort. An extension of this simplified framework to turbulent flow regime is examined revisiting the widely benchmarked flow at Reynolds number $R e=22000$, the theoretical predictions obtained in the frame of unsteady Reynoldsaveraged Navier-Stokes modeling being consistent with experimental data from the literature. Application of the various sensitivity frameworks to alternative control objectives such as increasing the lift and reducing the fluctuating drag and lift is also discussed and illustrated with a few selected examples. ( 2014 AIP Publishing LLC. [http://dx.doi.org/10.1063/1.4896941]
\end{abstract}

\section{INTRODUCTION}

The seminal analysis of Strykowski and Sreenivasan ${ }^{1}$ provides experimental evidence that a small circular cylinder positioned in the near wake of a main cylinder can alter vortex shedding at Reynolds numbers $R e \sim 50-100$ (based on the cylinder diameter and the free stream velocity) closely above the first instability threshold. For specific locations of this control cylinder, the authors find indeed a stabilization of the wake accompanied by a decrease of the shedding frequency that could go towards complete suppression of unsteadiness. Since then, similar results have been obtained from direct numerical simulation ${ }^{2,3}$ and global stability analysis ${ }^{4}$ of the two-cylinder system performed at about the same Reynolds numbers. The effect upon aerodynamic forces has been studied experimentally and numerically by Dalton, $\mathrm{Xu}$, and $\mathrm{Owen}^{5}$ and Yildirim, Rindt, and Steenhoven, ${ }^{6}$ who report reduction of the time-averaged mean drag and of the fluctuating lift, as well as enhancement of the mean lift at larger but still moderate Reynolds numbers ranging from 100 to 3000. Experimentally, the control cylinder technique has proven successful up to high, turbulent Reynolds numbers of order $10^{4}-10^{5}$; see Refs. $7-11$ applying the methodology to circular, square, 
and D-shaped geometries of the main cylinder. In these studies, varying the position of the control cylinder yields either an increase or a decrease of the shedding frequency, but shedding itself would not be extinguished on behalf of the large Reynolds number, hence constituting noticeable differences with regards to the analysis of Strykowski and Sreenivasan. ${ }^{1}$ A maximum drag reduction by $20 \%-30 \%$ is achieved (depending on the geometry and the Reynolds number) with either frequency increase or decrease. Cadot, Thiria, and Beaudoin ${ }^{12}$ assessed the ability of a second control cylinder (i.e., a third cylinder) in further increasing the base pressure of the D-shaped cylinder, hence resulting in additionally reduced drag.

Strykowski and Sreenivasan ${ }^{1}$ present their results in terms of sensitivity maps showing regions close to the main cylinder where shedding is most affected by the control cylinder. In the same vein, Sakamoto, Tan, and Haniu, ${ }^{7}$ Sakamoto and Haniu, ${ }^{8}$ and Parezanović and Cadot $^{11}$ map global quantities (e.g., Strouhal number, mean or root mean square values of drag and lift), the cost of which rapidly becomes prohibitive since systematical experimental measurements, numerical simulations or stability analyses must be performed over large parameter spaces including chiefly the position and diameter of the control cylinder (to give a taste, the Strouhal number map documented in Parezanović and $\mathrm{Cadot}^{11}$ is made assembling shedding frequencies measured at $\sim 5000$ sampled positions of the control cylinder). Actually, only a limited number of positions (of about a few ten) is considered in all other aforementioned studies, hence providing only an undersampled estimate of the real optimal. As an illustration, Dalton et al. ${ }^{5}$ fix the gap distance separating the centers of the two cylinders to 1.4 diameters of the main cylinder, then vary only the angle of attack (that is, the angle between the center-to-center line and the free stream direction) and report a maximum drag reduction by $33 \%$ in flow past a circular cylinder at $R e=100$. For the exact same flow case, Yildirim et al. ${ }^{6}$ fix the stream-wise position of the control cylinder to 0.75 diameter of the main cylinder, then vary only its cross-wise position and report a maximum drag reduction by only $6.5 \%$. The discrepancy of course arises from both groups of authors having spanned different near-wake regions, and motivates the development of more systematical approaches relying on theoretical analysis to map quickly the best positions for placement of the control cylinder.

The experiment of Strykowski and Sreenivasan ${ }^{1}$ has been revisited by Hill ${ }^{13}$ and subsequently by Marquet, Sipp, and Jacquin, ${ }^{14}$ using linear analysis to assess the effect of an infinitely small such control cylinder upon the stability of the main cylinder flow. First, the authors perform a global stability analysis of the uncontrolled base solution and determine the growth rate and eigenfrequency of the shedding eigenmode, i.e., the instability mode responsible for the onset of vortex shedding. ${ }^{15,16}$ Second, they calculate the linear eigenvalue variation induced by the control from the inner product between a sensitivity function (obtained with the adjoint method, and mathematically representing the variational derivative of the eigenvalue to sources of momentum in the flow field) and a body force mimicking the presence of the control cylinder. Finally, they determine flow regions of interest where the so-modeled cylinder would stabilize the shedding eigenmode, or decrease its eigenfrequency, their theoretical maps matching well the hard-earned ones of Strykowski and Sreenivasan. ${ }^{1}$ The approach offers an attractive alternative to bottleneck "trial and error" procedures in that it allows to span quickly all possible positions of the control cylinder without ever calculating the actually controlled states. It has led a substantial body of recent work focusing on steady and unsteady effects modeling the presence of the control cylinder, ${ }^{17-20}$ and is now applied to a variety of laminar flows at moderate Reynolds numbers ${ }^{21-25}$ as a means to gain beforehand valuable information regarding the most sensitive regions for open-loop control based on the underlying physics. Recently, Meliga, Pujals, and Serre ${ }^{26}$ have pushed forward the development of the method to turbulent flow regime generalizing the computation of the sensitivity function in the frame of unsteady Reynolds-averaged Navier-Stokes modeling. The authors determine the shedding eigenmode from a global stability analysis of the uncontrolled mean flow and identify specific regions where the control cylinder would either increase or decrease its eigenfrequency in flow past a D-shaped cylinder at $R e=13000$, in satisfactory agreement with the experiments of Parezanović and Cadot. ${ }^{11}$ As an illustration of the potential of the approach, the largest theoretical variations are obtained placing the control cylinder upstream of the main cylinder, in a flow region that has been precisely overlooked in the experiments. Since then, similar results have been reported by Mettot, Sipp, and Bezard ${ }^{27}$ using a simplified approach based on quasi-laminar analysis. 
The present research aims at assessing similarly the effect upon the aerodynamic forces. The main focus is on reducing the drag on a square cylinder, intended to serve as a testbed for development of the methodology, but we also discuss application to alternative control objectives such as increasing the lift and reducing the fluctuating drag and lift with a few selected examples. Following Hill, ${ }^{13}$ we estimate the drag variation induced by an infinitely small control cylinder from the inner product between relevant drag sensitivity functions computed with the adjoint method and a model reacting force localized at the same location where the control cylinder is placed, equal and opposite to the anticipated drag. The sensitivity functions are derived analytically building on previous work in the fields of shape optimization, ${ }^{28}$ inverse design, ${ }^{29}$ and adaptive mesh refinement. ${ }^{30,31}$ The numerical behaviour of the related adjoint equations has been discussed recently by Wang and $\mathrm{GaO}^{32}$ who evidenced amplification of adjoint kinetic energy along specific eigen-directions of the flow shear rate tensor. Nonetheless, the use of such theoretical frameworks to design flow control means for drag reduction purposes is reported here for the first time, to the best of the authors' knowledge. Since the control force modifies concurrently the growth rate of flow disturbances, ${ }^{1,13}$ we pay attention not to shift a steady (resp., an unsteady) natural—i.e., uncontrolled—flow into an unsteady (resp., a steady) controlled flow and perform our analysis only at Reynolds numbers not too close to the critical value $R e_{\mathrm{c}} \sim 50$ for the onset of a Hopf bifurcation into time-periodic limit cycle oscillations. ${ }^{15,33-35} \mathrm{We}$ thus consider three different cases, namely, a laminar steady case at Reynolds number $\operatorname{Re}=40$, a laminar unsteady case at $R e=100$, and a turbulent case at $R e=22000$. At $R e=40$, we examine the sensitivity of the steady asymptotic value of drag reached after the initial transient, which we show requires solving a steady adjoint problem from knowledge of only the stable base solution. At $R e=100$, we rather examine the sensitivity of the time-averaged mean drag, which we show requires solving an unsteady adjoint problem from knowledge of the entire history of the vortexshedding solution. We also introduce a novel alternative framework meant to approximate the mean drag sensitivity from a steady adjoint problem requiring knowledge of only the mean cylinder flow. As will be shown, the results obtained doing so are in quite good agreement with those obtained solving the exact unsteady adjoint problem, meaning that the approach carries valuable information in view of guiding efficient control strategy, besides reducing tremendously the numerical effort. In closing the study, we thus push forward the development of this simplified framework to turbulent flow regime revisiting the widely benchmarked Reynolds number $R e=22000$ and generalizing the computation of the related sensitivity in the frame of unsteady Reynolds-averaged Navier-Stokes modeling.

The manuscript is organized as follows. The flow configuration is described in Sec. II. The theoretical frameworks for laminar steady and unsteady flow regimes are presented in Secs. III and IV, including derivation of the sensitivity of (steady asymptotic or mean) drag with the adjoint method, application to open-loop control by means of a small control cylinder, and comparison with numerical simulations of the two-cylinder system for selected positions of interest. The simplified framework for laminar unsteady flow regime is introduced in Sec. V, and the results thereof are compared to the exact ones documented in Sec. IV. The extension to turbulent flow regime is considered in Sec. VI.

\section{FLOW CONFIGURATION}

Two-dimensional (2D) laminar incompressible flow past a span-wise infinite square cylinder subjected to a uniform stream at zero incidence is considered a prototype of wake past a slender body. The flow is described in a Cartesian coordinate system $(x, y)$ with drag force positive in the stream-wise $+x$ direction and lift force positive in the cross-wise $+y$ direction; see Fig. 1 . The origin is at the center of the cylinder, which has diameter $d^{*}$. Constant density $\rho^{*}$ and kinematic viscosity $v^{*}$ is assumed, therefore the sole parameter for this problem is the Reynolds number $R e=u_{\infty}^{*} d^{*} / v^{*}$, with $u_{\infty}^{*}$ being the free-stream velocity. The velocity vector is $\boldsymbol{u}=(u, v)$ with $u$ and $v$ the stream-wise and cross-wise components. Pressure is denoted by $p$. In the following, all variables are non-dimensionalized with respect to the cylinder diameter and the free-stream velocity. The evolution of the fluid flow in space domain $\Omega$ is governed by the Navier-Stokes 


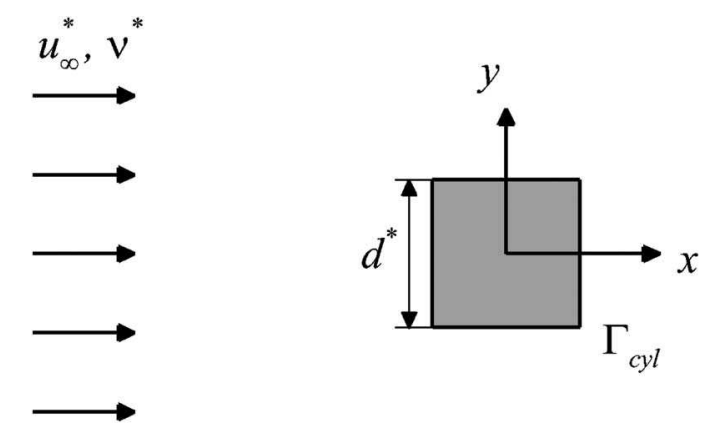

FIG. 1. Schematic diagram of the square cylinder configuration.

equations

$$
\begin{aligned}
& \boldsymbol{\nabla} \cdot \boldsymbol{u}=\mathbf{0}, \quad \partial_{t} \boldsymbol{u}+\boldsymbol{\nabla} \boldsymbol{u} \cdot \boldsymbol{u}-\nabla \cdot \boldsymbol{\sigma}(p, \boldsymbol{u})=\mathbf{0} \quad \text { in } \Omega, \\
& \boldsymbol{u}=\mathbf{0} \quad \text { on } \Gamma_{\text {cyl }},
\end{aligned}
$$

where $\Gamma_{\text {cyl }}$ denotes the cylinder surface and $\sigma$ is the stress tensor

$$
\boldsymbol{\sigma}(p, \boldsymbol{u})=-p \boldsymbol{I}+\frac{1}{R e}\left(\nabla \boldsymbol{u}+\nabla \boldsymbol{u}^{\mathrm{T}}\right) .
$$

Numerically, this problem, as well as those formulated below, is subject to appropriate open flow conditions on the outer boundary $\partial \Omega$ of the space domain; see Secs. III B and IV B for further details.

The present study aims at assessing the effect of a control force $\delta \boldsymbol{f}$ upon the resultant force on the cylinder $D \boldsymbol{e}_{\boldsymbol{x}}+L \boldsymbol{e}_{\boldsymbol{y}}$, where $D$ and $L$ are the drag and lift coefficients per unit length (simply termed drag and lift to ease the reading) defined as

$$
D=2 \oint_{\Gamma_{\mathrm{cyl}}}\{\boldsymbol{\sigma}(p, \boldsymbol{u}) \cdot \boldsymbol{n}\} \cdot \boldsymbol{e}_{\boldsymbol{x}} \mathrm{d} l \quad \text { and } \quad L=2 \oint_{\Gamma_{\mathrm{cy} 1}}\{\boldsymbol{\sigma}(p, \boldsymbol{u}) \cdot \boldsymbol{n}\} \cdot \boldsymbol{e}_{\boldsymbol{y}} \mathrm{d} l,
$$

and $\boldsymbol{n}$ is the unit outward normal at the cylinder surface. As long as the intending meaning is clear from the context, we will use cylinder flow to refer either to the natural cylinder flow, i.e., the solution to Eq. (1), or to the controlled cylinder flow, i.e., the solution to the same equations with body force $\delta f$ as an additional right-hand side.

\section{STEADY SENSITIVITY ANALYSIS IN LAMINAR STEADY FLOW REGIME}

Only steady control forces are considered in this section. The quantity of interest is the steady asymptotic drag

$$
D=2 \oint_{\Gamma_{\mathrm{cyl}}}\left\{\boldsymbol{\sigma}\left(p_{b}, \boldsymbol{u}_{b}\right) \cdot \boldsymbol{n}\right\} \cdot \boldsymbol{e}_{\boldsymbol{x}} \mathrm{d} l,
$$

where we denote for clarity by $\left(\boldsymbol{u}_{b}, p_{b}\right)$ the natural base flow, solution of the steady Navier-Stokes equations

$$
\begin{array}{rll}
\nabla \cdot \boldsymbol{u}_{b}=\mathbf{0}, & \boldsymbol{\nabla} \boldsymbol{u}_{b} \cdot \boldsymbol{u}_{b}-\nabla \cdot \boldsymbol{\sigma}\left(p_{b}, \boldsymbol{u}_{b}\right)=\mathbf{0} & \text { in } \Omega, \\
\boldsymbol{u}_{b}=\mathbf{0} & \text { on } \Gamma_{\mathrm{cyl}}
\end{array}
$$

\section{A. Theoretical framework}

The change in drag induced by an infinitesimal control force $\delta \boldsymbol{f}$ is expressed as the inner product between a sensitivity function (representing the variational derivative of the steady asymptotic drag to sources of momentum in the flow) and the control force itself. This amounts to invoking the first-order Taylor expansion around zero of $D$ viewed as a function of $\delta \boldsymbol{f}$, given that the sensitivity 
depends on the choice of the inner product structure, but that the variation computed from the inner product does not. We seek here the sensitivity function $\nabla_{f} D$ such that

$$
\delta D=2 \oint_{\Gamma_{\mathrm{cyl}}}\left\{\boldsymbol{\sigma}\left(\delta p_{b}, \boldsymbol{\delta} \boldsymbol{u}_{b}\right) \cdot \boldsymbol{n}\right\} \cdot \boldsymbol{e}_{\boldsymbol{x}} \mathrm{d} l=\left(\nabla_{f} D \mid \boldsymbol{\delta} \boldsymbol{f}\right),
$$

where $\left(\delta \boldsymbol{u}_{b}, \delta p_{b}\right)$ is the base flow modification, i.e., the linear perturbation to the base solution induced by the control force through the steady, linear direct system

$$
\begin{aligned}
\boldsymbol{\nabla} \cdot \boldsymbol{\delta} \boldsymbol{u}_{b}=\mathbf{0}, \quad \boldsymbol{\nabla} \boldsymbol{\delta} \boldsymbol{u}_{b} \cdot \boldsymbol{u}_{b}+\nabla \boldsymbol{u}_{b} \cdot \boldsymbol{\delta} \boldsymbol{u}_{b}-\nabla \cdot \boldsymbol{\sigma}\left(\delta p_{b}, \boldsymbol{\delta} \boldsymbol{u}_{b}\right)=\boldsymbol{\delta} \boldsymbol{f} & \text { in } \Omega, \\
\boldsymbol{\delta} \boldsymbol{u}_{b}=\mathbf{0} & \text { on } \Gamma_{\mathrm{cyl}},
\end{aligned}
$$

and we denote by ( | ) the $L_{2}$ inner product on the space domain $\Omega$, i.e.,

$$
(\boldsymbol{a} \mid \boldsymbol{b})=\int_{\Omega} \boldsymbol{a} \cdot \boldsymbol{b} \mathrm{d} x \mathrm{~d} y .
$$

Note the first equality in (6) follows from the linearity of drag in the flow quantities. Note also we compute only the inner product of real-valued quantities, so complex conjugation does not appear in (6)-(8).

An analytical expression for the sensitivity function is derived with a variational technique based on the computation of Lagrange multipliers, as in classical optimization problems. ${ }^{36}$ We obtain

$$
\nabla_{f} D=u_{b}^{\dagger}
$$

where we denote by $\left(\boldsymbol{u}_{b}^{\dagger}, p_{b}{ }^{\dagger}\right)$ the solution of the steady, linear, adjoint system

$$
\begin{aligned}
& \boldsymbol{\nabla} \cdot \boldsymbol{u}_{b}^{\dagger}=\mathbf{0}, \quad-\boldsymbol{\nabla} \boldsymbol{u}_{b}^{\dagger} \cdot \boldsymbol{u}_{b}+\nabla \boldsymbol{u}_{b}{ }^{\top} \cdot \boldsymbol{u}_{b}^{\dagger}-\boldsymbol{\nabla} \cdot \boldsymbol{\sigma}\left(-p_{b}^{\dagger}, \boldsymbol{u}_{b}^{\dagger}\right)=\mathbf{0} \text { in } \Omega, \\
& \boldsymbol{u}_{b}^{\dagger}=2 \boldsymbol{e}_{\boldsymbol{x}} \text { on } \Gamma_{\mathrm{cyl}},
\end{aligned}
$$

consisting of homogeneous equations of motion and non-homogeneous boundary conditions. The rigorous mathematical proof is performed in Appendix B 1, but in short, we craft Eq. (10) to be adjoint to the steady linearized Navier-Stokes equations via integration by parts and the divergence theorem, and make the bilinear concomitant on $\partial \Omega$ be zero by an appropriate choice of adjoint boundary conditions. We show in Appendix B 1 that this yields ultimately

$$
\int_{\Omega} \boldsymbol{u}_{b}^{\dagger} \cdot \boldsymbol{\delta} \boldsymbol{f} \mathrm{d} x \mathrm{~d} y=\oint_{\Gamma_{\mathrm{cyl}}}\left\{\boldsymbol{\sigma}\left(\delta p_{b}, \delta \boldsymbol{u}_{b}\right) \cdot \boldsymbol{n}\right\} \cdot \boldsymbol{u}_{b}^{\dagger} \mathrm{d} l,
$$

where the right-hand side in (11) is the remaining bilinear concomitant on $\Gamma_{\text {cyl }}$, and relation (9) follows straightforwardly replacing $\boldsymbol{u}_{b}^{\dagger}$ by its boundary value $2 \boldsymbol{e}_{\boldsymbol{x}}$. It is worth insisting that relation (11) is only formal, in the sense that we use knowledge of the direct system (7) to set up the adjoint framework, but we do not need knowledge of the direct solution $\left(\boldsymbol{\delta} \boldsymbol{u}_{b}, \delta p_{b}\right)$ to solve the adjoint system (10). This, of course, is where lies much of the power of the approach.

\section{B. Numerical method}

The numerical approach used to solve the various problems of interest is adapted from Meliga and $\mathrm{Chomaz}^{20}$ to which the reader is referred for further details. The $2 \mathrm{D}$ computational domain is defined as

$$
\Omega=\left\{(x, y) \mid \max (|x|,|y|) \geq 0.5 ; x_{-\infty} \leq x \leq x_{\infty} \text { and }|y| \leq y_{\infty}\right\},
$$

where the values $x_{-\infty}=-100, x_{\infty}=150$, and $y_{\infty}=25$ are chosen large enough not to have a discernible influence on the results. A mesh composed of triangular elements is generated using the Delaunay-Voronoi algorithm with strong clustering at the cylinder surface and in the near wake. The finite-element FreeFem ++ software $^{37}$ is used to discretize all differential operators on a mixed base of Arnold-Brezzi-Fortin MINI-elements (piecewise linear continuous finite elements with three degrees of freedom at each triangle edge for pressure, same element enriched with a cubic bubble 


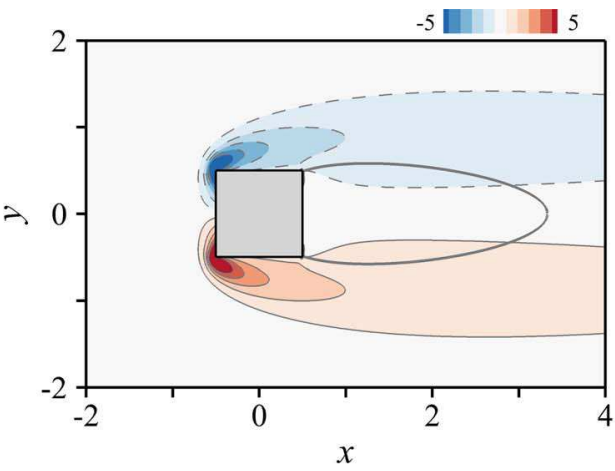

(a)

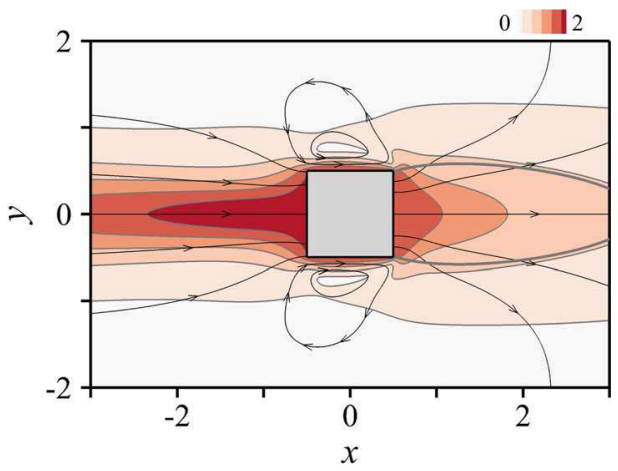

(b)

FIG. 2. Square cylinder flow at $R e=40$. (a) Iso-contours of the base vorticity. The thick gray line is the separatrix of the recirculation region. (b) Spatial distribution of the steady asymptotic drag sensitivity. The magnitude of sensitivity is given by the color levels and the orientation of the underlying vector by the superimposed streamlines.

function at the barycenter of each triangle for velocity components). The resulting linear systems are solved with the sparse direct LU solver embedded in the UMFPACK library. ${ }^{38,39}$

We compute first the natural base solution using the iterative Newton-Raphson method, with open flow boundary conditions consisting of a uniform free-stream $\boldsymbol{u}_{b}=\boldsymbol{e}_{\boldsymbol{x}}$ at the inflow, symmetric conditions $\partial_{y} u_{b}=v_{b}=0$ at the transverse boundaries and a stress-free condition $\boldsymbol{\sigma}\left(p_{b}, \boldsymbol{u}_{b}\right) \cdot \boldsymbol{n}=\mathbf{0}$ at the outflow. If we let the direct solution (albeit not computed in practice) satisfy conditions linearized from the above ones, namely $\delta \boldsymbol{u}_{b}=\mathbf{0}$ at the inlet, $\partial_{y} \delta u_{b}=\delta v_{b}=0$ at the transverse boundaries and $\boldsymbol{\sigma}\left(\delta p_{b}, \boldsymbol{\delta} \boldsymbol{u}_{b}\right) \cdot \boldsymbol{n}=\mathbf{0}$ at the outflow, we then solve the adjoint system with homogeneous conditions $\boldsymbol{u}_{b}^{\dagger}=\mathbf{0}$ at the inflow, symmetric conditions $\partial_{y} u_{b}{ }^{\dagger}=v_{b}{ }^{\dagger}=0$ at the transverse boundaries and an adjoint stress-free condition $\boldsymbol{\sigma}\left(-p_{b}^{\dagger}, \boldsymbol{u}_{b}^{\dagger}\right) \cdot \boldsymbol{n}+\left(\boldsymbol{u}_{b} \cdot \boldsymbol{n}\right) \boldsymbol{u}_{b}^{\dagger}=\mathbf{0}$ at the outflow to make the bilinear concomitant on $\partial \Omega$ be zero; see details in Appendix B 1. All results reported in this section pertain to the same reference mesh made of 792452 triangles (2 765452 degrees of freedom in terms of a velocity-pressure vector). Grid independence has been checked comparing to two other grid resolutions and spatial extents, which led a change in the numerical values by less than $0.1 \%$. The correctness and numerical accuracy of the adjoint calculations have been assessed from systematical validation tests documented in Appendix B 2.

The Reynolds number is set to $R e=40$ in the remainder of the section. Typical contours of the base vorticity are shown in Fig. 2(a). The distribution is antisymmetric with respect to the centreline $y=0$. Two shear layers displaying vorticity of opposite signs form at the upstream stagnation point and develop over the front side of the cylinder. They turn around the leading edges (where the magnitudes of vorticity are the largest) as they remain attached on the top/bottom sides, and ultimately separate at the trailing edges. The so-formed recirculation extends 2.83 cylinder diameters downstream of the cylinder base, as evidenced by the thick gray line representing the separating streamline. The drag computed from Eq. (3) is $D=1.67$, which compares well with existing data. ${ }^{40}$ The sensitivity function is depicted in Fig. 2(b). Streamlines of the underlying vector field give the local orientation of the gradient, and color levels indicate its magnitude. In practice, a local force $\boldsymbol{\delta} \boldsymbol{f}$ oriented in the same direction (resp., in the opposite direction) as the arrows plotted in Fig. 2(b) therefore increases (resp., decreases) drag by a quantity proportional to the local magnitude. The maximum magnitude of sensitivity is reached approximately one diameter upstream of the cylinder, but significant levels persist further upstream, up to several tens of diameters. The magnitude is large also in the shear layers and in the near wake, including the recirculation region, and decays slowly as the vorticity diffuses in the far wake.

\section{Application to open-loop control by a small circular cylinder}

We now use knowledge of the sensitivity as a systematic guideline on where to insert a small device in the attempt to reduce the steady asymptotic drag. We consider a small circular control 


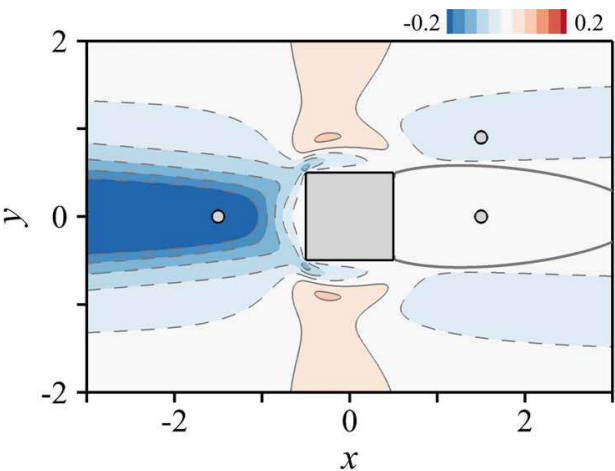

(a)

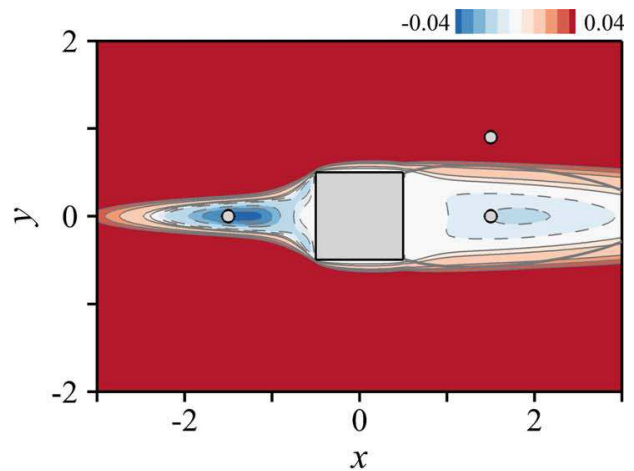

(b)

FIG. 3. Variation of steady asymptotic drag induced by a control cylinder of diameter $\eta=0.1$ whose presence is modeled by Eqs. (13) and (14) - $R e=40$. (a) Drag of the square cylinder. (b) Drag of the two-cylinder system. For both plots, the various circle symbols mark the positions for which the theoretical predictions are compared to base flow calculations of open-loop control by a small control cylinder in Fig. 4.

cylinder of diameter $\eta$, whose presence at given position $\left(x_{\mathrm{c}}, y_{\mathrm{c}}\right)$ is modeled by the force it exerts on the flow, expressed as

$$
\boldsymbol{\delta} \boldsymbol{f}_{\eta}(x, y)=-\frac{1}{2} \eta D_{\eta}\left(R e_{\eta}\right) \boldsymbol{\xi}\left(x_{\mathrm{c}}, y_{\mathrm{c}}\right) \delta\left(x-x_{\mathrm{c}}, y-y_{\mathrm{c}}\right) .
$$

In (13), $\boldsymbol{\xi}=\|\boldsymbol{u}\| \boldsymbol{u}$ is the signed square velocity vector $(\|\cdot\|$ being the norm induced by the dot product), and $D_{\eta}$ is the drag coefficient of the control cylinder, whose dependency on the local Reynolds number $R e_{\eta}=\eta u_{\mathrm{c}} R e$ (based on the diameter of the control cylinder and the magnitude of the local velocity $\left.u_{\mathrm{c}}=\left\|\boldsymbol{u}_{b}\left(x_{\mathrm{c}}, y_{\mathrm{c}}\right)\right\|\right)$ is approximated using a three-parameter power law

$$
D_{\eta}\left(R e_{\eta}\right)=a_{0}+a_{1} R e_{\eta}{ }^{a_{2}} .
$$

We use values $a_{0}=0.8558, a_{1}=10.05$, and $a_{2}=-0.7084$ meant to fit data from the literature ${ }^{41,42}$ and in-house numerical data for steady flow in a range $R e_{\eta}<20$ relevant for small but noninfinitesimal diameters up to $\eta \sim 0.4$, since we recall that the Reynolds number is bounded from above by its critical value $R e_{\mathrm{c}}=50$. Equation (13) defines $\delta f_{\eta}$ as a reacting force localized at the same location where the control cylinder is placed, equal and opposite to the force that would act on a fictitious cylinder of same diameter subjected to a uniform flow at the local steady flow velocity. Note we have anticipated the latter force to be pure steady drag on behalf of the low values of $R e_{\eta}$. The present framework is thus relevant in examining the effect of the reacting force whose amplitude can be checked to go to zero as $\eta$ goes to zero.

We map in Fig. 3(a) the variation of the cylinder drag $\delta D$ induced by a control cylinder of diameter $\eta=0.1$ at $R e=40$, for which the local Reynolds number is below 5. To each position $\left(x_{\mathrm{c}}, y_{\mathrm{c}}\right)$ of the control cylinder is associated a drag variation computed as

$$
\delta D\left(x_{\mathrm{c}}, y_{\mathrm{c}}\right)=\left(\boldsymbol{u}_{b}^{\dagger} \mid \boldsymbol{\delta} \boldsymbol{f}_{\eta}\right)=-\frac{1}{2} \eta D_{\eta} \boldsymbol{u}_{b}^{\dagger}\left(x_{\mathrm{c}}, y_{\mathrm{c}}\right) \cdot \boldsymbol{\xi}\left(x_{\mathrm{c}}, y_{\mathrm{c}}\right) .
$$

The map is symmetric with respect to the centreline $y=0$ and exhibits both negative and positive variations corresponding respectively to a decrease (as indicated by the blue hue) or an increase (red hue) of the cylinder drag. It indicates that there are two main flow regions in which the cylinder drag is reduced, a large one upstream of the cylinder extending over 10 diameters of the main cylinder or so, and another one extending downstream along the outer boundary of the recirculation region. A maximum reduction by $20 \%$ is achieved placing the control cylinder in the upstream region. Moving away from the cylinder surface in the cross-wise direction, the drag decreases first in a narrow strip originating from the leading edges, but subsequently increases in a larger region extending up to the potential flow. Fig. 3(b) proposes a map of the total drag variation

$$
\delta D_{\mathrm{tot}}\left(x_{\mathrm{c}}, y_{\mathrm{c}}\right)=\frac{1}{2} \eta D_{\eta}\left(\boldsymbol{u}_{b}^{\dagger}\left(x_{\mathrm{c}}, y_{\mathrm{c}}\right)-2 \boldsymbol{e}_{\boldsymbol{x}}\right) \cdot \boldsymbol{\xi}\left(x_{\mathrm{c}}, y_{\mathrm{c}}\right),
$$




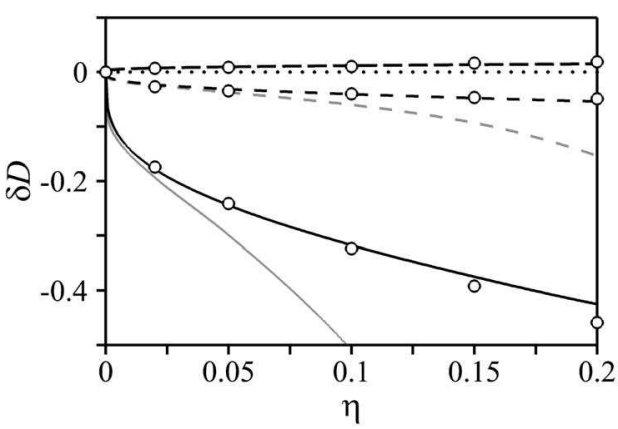

(a)

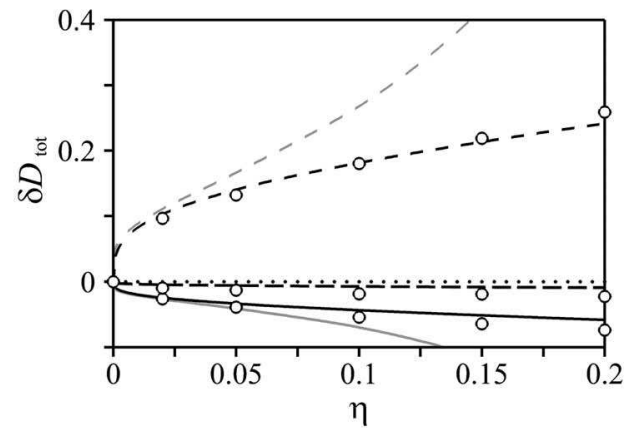

(b)

FIG. 4. Variation of steady asymptotic drag as a function of the diameter of the control cylinder at $R e=40$. The black lines denote theoretical predictions obtained modeling the presence of the cylinder at positions $\left(x_{\mathfrak{c}}, y_{\mathrm{c}}\right)=(-1.5,0)$ (solid line), $\left(x_{\mathrm{c}}, y_{\mathrm{c}}\right)=(1.5,0.9)$ (dashed line), and $\left(x_{\mathrm{c}}, y_{\mathrm{c}}\right)=(1.5,0)$ (long-dashed line) by Eq. (13) and approximating the dependence on Reynolds number of the drag coefficient $D_{\eta}$ by the three-parameter power law (14). The gray lines denote alternative predictions obtained using the Oseen drag formula (19) for circular cylinder in Stokes flow. The exact values obtained from base flow computation of flow over the two cylinders are superimposed as the open symbols. (a) Drag of the cylinder. (b) Drag of the two-cylinder system.

representing the variation between the drag of the two-cylinder system and that of the natural cylinder flow, to be considered a measure of the control net efficiency. It indicates that there is essentially one region left in which the total drag is reduced, namely the upstream region of largest sensitivity. However, its spatial extent is limited to 2-3 cylinder diameters with maximum achieved reduction by 5\%. In all other regions of interest identified from Fig. 3(a), the drag of the main cylinder is not sufficiently reduced to compensate for the fact that the control cylinder itself is a source of drag. Note the total drag is slightly reduced in the recirculation region despite small positive values of $\delta D$, which is because the stream-wise velocity is negative in this region and makes the control cylinder be a source of thrust, not drag.

\section{Comparison with steady asymptotic drag of the two-cylinder system}

The black lines in Fig. 4 stand for the variations $\delta D$ and $\delta D_{\text {tot }}$ computed from Eqs. (15) and (16), placing the control cylinder at the three positions marked by the symbols in Fig. 3 and varying its diameter up to $\eta=0.2$. The solid line is for the first position $\left(x_{\mathrm{c}}, y_{\mathrm{c}}\right)=(-1.5,0)$ where the control cylinder decreases both the cylinder and the total drag, the dashed line is for the second position $\left(x_{\mathrm{c}}, y_{\mathrm{c}}\right)=(1.5,0.9)$ where it decreases the cylinder drag but increases the total drag, and the longdashed line is for the third position $\left(x_{\mathrm{c}}, y_{\mathrm{c}}\right)=(1.5,0)$ where it increases the cylinder drag but decreases the total drag, consistently with the results of Fig. 3. The superimposed symbols represent numerical data points of open-loop control by means of a small control cylinder, i.e., each point is obtained meshing the computational domain of the two-cylinder system

$$
\Omega_{\eta}=\left\{(x, y) \mid \max (|x|,|y|) \geq 0.5 ; \sqrt{\left(x-x_{\mathrm{c}}\right)^{2}+\left(y-y_{\mathrm{c}}\right)^{2}} \geq \eta ; x_{-\infty} \leq x \leq x_{\infty} \text { and }|y| \leq y_{\infty}\right\},
$$

and using the Newton-Raphson method to solve the steady Navier-Stokes equations

$$
\begin{aligned}
\nabla \cdot \boldsymbol{u}_{b}=\mathbf{0}, \quad \nabla \boldsymbol{u}_{b} \cdot \boldsymbol{u}_{b}-\nabla \cdot \boldsymbol{\sigma}\left(p_{b}, \boldsymbol{u}_{b}\right)=\mathbf{0} & \text { in } \Omega, \\
\boldsymbol{u}_{b}=\mathbf{0} & \text { on } \Gamma_{\text {cyl }} \cup \Gamma_{\eta},
\end{aligned}
$$

where $\Gamma_{\eta}$ denotes the surface of the control cylinder. In the range $0.02 \leq \eta \leq 0.2$ of interest, specific grid refinement tests show that the flow is accurately represented distributing 300 points at the surface of the control cylinder. In return, not only the obtained results follow the theoretical trends, but a striking agreement is observed for all values of $\eta$ considered, hence demonstrating the ability of the approach in providing qualitative and quantitative predictions. The limit of validity of the small control cylinder assumption can be estimated to a first approximation taking $\eta$ to be smaller by 
a factor of 10 than both the diameter of the main cylinder and the local inhomogeneity length scale (as measured by the ratio of the magnitude of local velocity to the magnitude of the local velocity gradient). This yields values in the range $0.05-0.1$, meaning that the model actually keeps providing relevant predictions even though the assumptions underlying its derivation may not be rigorously satisfied. We believe this is because our analytical law (14) is calibrated numerically to reproduce the effect of a small but finite control cylinder. As an illustration, we report a second set of adjoint-based predictions (shown as the gray lines in Fig. 4) obtained approximating the dependence on Reynolds number of the drag coefficient with the Oseen drag formula for circular cylinder in Stokes flow,

$$
D_{\eta}\left(R e_{\eta}\right)=\frac{8 \pi}{R e_{\eta}}\left(\log \frac{8}{R e_{\eta}}-\gamma+\frac{1}{2}\right)^{-1},
$$

where $\gamma$ is the Euler constant. Both laws (14)-(19) are meant to fit the same reference values of $D_{\eta}$ in the limit as $\eta$ (and thereby $R e_{\eta}$ ) goes to zero, where the Oseen formula is rigorously valid. Therefore, the agreement between both sets of theoretical results at the smallest values of $\eta$ is not a surprise. The agreement keeps being very satisfactory for all values of $\eta$ considered at $\left(x_{\mathrm{c}}, y_{\mathrm{c}}\right)$ $=(1.5,0)$ because the local Reynolds number is very small $\left(R e_{\eta}<0.7\right)$ owing to the low magnitude of the local velocity in the recirculation region. For the other two positions, the agreement starts being only in order of magnitudes above a threshold diameter in a range $0.03-0.05$ consistent with the value $1 /\left(u_{\mathrm{c}} R e\right)$ for which the local Reynolds number is unity (which is approximately the limit of validity of the Oseen formula). These results clearly demonstrate that the sensitivity provides a systematic path to guide the placement of the control cylinder in the sense that the localization of the sensitive regions can be inferred with good accuracy even though the degree of approximation used to represent the control cylinder itself is quite poor. In contrast, they clearly stress the need for a higher degree of approximation to capture quantitatively the effect of a finite size cylinder.

\section{E. Effect upon the steady asymptotic lift}

Only minor modification to the above theoretical framework is required to assess the effect of the control cylinder upon the steady asymptotic lift, as we show in Appendix B 3 that the related sensitivity function is simply

$$
\nabla_{f} L=\boldsymbol{u}_{b}^{\dagger}
$$

where $\left(\boldsymbol{u}_{b}^{\dagger}, p_{b}^{\dagger}\right)$ denotes here the solution to the adjoint system

$$
\begin{array}{rr}
\nabla \cdot \boldsymbol{u}_{b}^{\dagger}=\mathbf{0}, \quad-\nabla \boldsymbol{u}_{b}^{\dagger} \cdot \boldsymbol{u}_{b}+\nabla \boldsymbol{u}_{b}{ }^{\mathrm{T}} \cdot \boldsymbol{u}_{b}^{\dagger}-\nabla \cdot \boldsymbol{\sigma}\left(-p_{b}^{\dagger}, \boldsymbol{u}_{b}^{\dagger}\right)=\mathbf{0} & \text { in } \Omega, \\
\boldsymbol{u}_{b}^{\dagger}=2 \boldsymbol{e}_{\boldsymbol{y}} & \text { on } \Gamma_{\mathrm{cyl}},
\end{array}
$$

differing from its drag counterpart (10) only by the boundary condition at the cylinder surface. In return, the lift variation computed from the model force (13) and mapped in Fig. 5 indicates that a control cylinder of diameter $\eta=0.1$ increases the steady asymptotic lift at $R e=40$ if positioned in the shear region originating from the lower leading edge and spreading on the cylinder length over the bottom side.

\section{UNSTEADY SENSITIVITY ANALYSIS IN LAMINAR UNSTEADY FLOW REGIME}

For given physical quantity $s$, we denote by $\bar{s}$ its time-averaged, mean value

$$
\bar{s}=\frac{1}{\tau} \int_{0}^{\tau} s \mathrm{~d} t,
$$

where $\tau$ is an averaging time-span assumed appropriately large enough to achieve convergence to statistical equilibrium, and by $s^{\prime}$ its fluctuation, by definition such that $s=\bar{s}+s^{\prime}$ and $\overline{s^{\prime}}=0$. The 


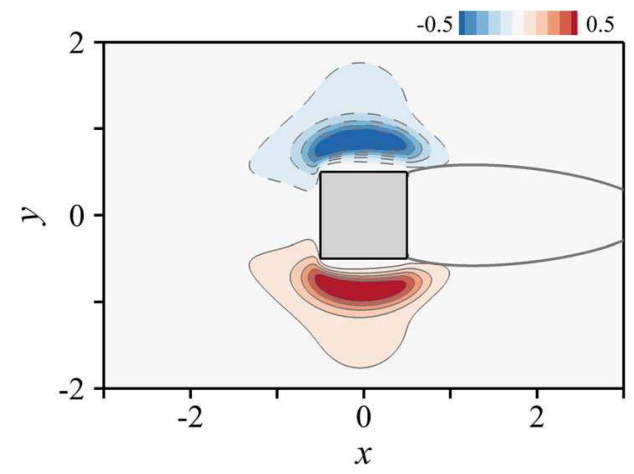

FIG. 5. Variation of steady asymptotic lift induced by a control cylinder of diameter $\eta=0.1$ whose presence is modeled by Eqs. (13) and (14) - $R e=40$.

quantity of interest in this section is thus the mean drag

$$
\bar{D}=\frac{2}{\tau} \int_{0}^{\tau} \oint_{\Gamma_{\mathrm{cyl}}}\{\boldsymbol{\sigma}(p, \boldsymbol{u}) \cdot \boldsymbol{n}\} \cdot \boldsymbol{e}_{\boldsymbol{x}} \mathrm{d} l \mathrm{~d} t,
$$

where $(\boldsymbol{u}, p)$ is the instantaneous cylinder flow, solution of the unsteady Navier-Stokes equations

$$
\begin{aligned}
\nabla \cdot \boldsymbol{u}=\mathbf{0}, & \partial_{t} \boldsymbol{u}+\nabla \boldsymbol{u} \cdot \boldsymbol{u}-\nabla \cdot \boldsymbol{\sigma}(p, \boldsymbol{u})=\mathbf{0} & & \text { in } \Omega, \\
\boldsymbol{u} & =\mathbf{0} & & \text { on } \Gamma_{\text {cyl }},
\end{aligned}
$$

with arbitrary initial condition at $t=0$.

\section{A. Theoretical framework}

As has been done in Sec. III, the change in drag $\overline{\delta D}$ induced by an infinitesimal control force $\delta \boldsymbol{f}$ is expressed as the inner product between a sensitivity function (now representing the variational derivative of the mean drag to sources of momentum in the flow) and the control force itself. We seek here the sensitivity function $\nabla_{f} \bar{D}$ such that

$$
\overline{\delta D}=\frac{2}{\tau} \int_{0}^{\tau} \oint_{\Gamma_{\mathrm{cyl}}}\{\boldsymbol{\sigma}(\delta p, \boldsymbol{\delta} \boldsymbol{u}) \cdot \boldsymbol{n}\} \cdot \boldsymbol{e}_{\boldsymbol{x}} \mathrm{d} l \mathrm{~d} t=\left(\left(\nabla_{\boldsymbol{f}} \bar{D} \mid \boldsymbol{\delta} \boldsymbol{f}\right)\right),
$$

where $(\delta \boldsymbol{u}, \delta p)$ is the instantaneous linear perturbation to the cylinder flow induced through the unsteady direct system

$$
\begin{array}{rll}
\nabla \cdot \delta \boldsymbol{u}=\mathbf{0}, & \partial_{t} \delta \boldsymbol{u}+\nabla \delta \boldsymbol{u} \cdot \boldsymbol{u}+\nabla \boldsymbol{u} \cdot \boldsymbol{\delta} \boldsymbol{u}-\nabla \cdot \boldsymbol{\sigma}(\delta p, \delta \boldsymbol{u})=\boldsymbol{\delta} \boldsymbol{f} & \text { in } \Omega, \\
\delta \boldsymbol{u}=\mathbf{0} & \text { on } \Gamma_{\text {cyl }}, \\
\delta \boldsymbol{u}=\mathbf{0} & \text { at } t=0,
\end{array}
$$

and we denote by ( $\mid$ | )) the tensorized $L_{2}$ inner product on the time-space domain $(0, \tau) \times \Omega$

$$
((\boldsymbol{a} \mid \boldsymbol{b}))=\frac{1}{\tau} \int_{0}^{\tau}(\boldsymbol{a} \mid \boldsymbol{b}) \mathrm{d} t
$$

consisting of an average over time of the $L_{2}$ spatial inner product used in Sec. III. Note the zero initial condition in (26) corresponding to arbitrary yet prescribed flow state a $t=0$.

The sensitivity function can be expressed analytically as

$$
\nabla_{f} \bar{D}=\boldsymbol{u}^{\dagger}
$$


where we denote by $\left(\boldsymbol{u}^{\dagger}, p^{\dagger}\right)$ the solution of the unsteady adjoint system

$$
\begin{aligned}
& \boldsymbol{\nabla} \cdot \boldsymbol{u}^{\dagger}=\mathbf{0}, \quad-\partial_{t} \boldsymbol{u}^{\dagger}-\nabla \boldsymbol{u}^{\dagger} \cdot \boldsymbol{u}+\nabla \boldsymbol{u}^{\mathrm{T}} \cdot \boldsymbol{u}^{\dagger}-\nabla \cdot \sigma\left(-p^{\dagger}, \boldsymbol{u}^{\dagger}\right)=\mathbf{0} \quad \text { in } \Omega, \\
& \boldsymbol{u}^{\dagger}=2 \boldsymbol{e}_{\boldsymbol{x}} \text { on } \Gamma_{\mathrm{cyl}}, \\
& \boldsymbol{u}^{\dagger}=\mathbf{0} \quad \text { at } t=\tau,
\end{aligned}
$$

to be solved backwards in time since time and directionality of advection by the cylinder flow are being reversed in (29), which, in practice, requires knowledge of the entire history of the cylinder flow solution through the time-span of interest. The rigorous mathematical proof performed in Appendix C 1 relies on a modified variational technique in which we craft problem (29) to be adjoint to the unsteady linearized Navier-Stokes equations via integration by parts and the divergence theorem, the zero adjoint "initial" condition at $t=\tau$ being intended to make the bilinear concomitant on $(0, \tau)$ be zero. We show in Appendix $\mathrm{C} 1$ that this yields ultimately

$$
\frac{1}{\tau} \int_{0}^{\tau} \int_{\Omega} \boldsymbol{u}^{\dagger} \cdot \boldsymbol{\delta} \boldsymbol{f} \mathrm{d} x \mathrm{~d} y \mathrm{~d} t=\frac{1}{\tau} \int_{0}^{\tau} \oint_{\Gamma_{\text {cyl }}}\{\boldsymbol{\sigma}(\delta p, \delta \boldsymbol{u}) \cdot \boldsymbol{n}\} \cdot \boldsymbol{u}^{\dagger} \mathrm{d} l \mathrm{~d} t,
$$

where the right-hand side in (30) is the remaining bilinear concomitant on $\Gamma_{\text {cyl }}$, and relation (28) follows straightforwardly replacing $\boldsymbol{u}^{\dagger}$ by its boundary value $2 \boldsymbol{e}_{\boldsymbol{x}}$. We insist again that Eq. (30) is only formal in the sense that we use knowledge of the direct system (26) to set up the adjoint framework, but we do not need knowledge of the direct solution $(\delta \boldsymbol{u}, \delta p)$ to solve the adjoint system (29).

\section{B. Numerical method}

The numerical approach is the same as described in Sec. III B with additional time discretization using the second-order Crank-Nicholson scheme. The dimensions of the computational domain reduce to $x_{-\infty}=-30, x_{\infty}=60$, and $y_{\infty}=25$. All results reported in this section pertain to the same reference mesh made of 111478 triangles (390 899 degrees of freedom) found to offer a good compromise between numerical accuracy and computational effort since numerical tests carried out at two other grid resolutions and spatial extents yield limited variations within $2 \%-3 \%$.

Direct numerical simulation (DNS) of the natural cylinder flow is performed with the same open flow boundary conditions as in Sec. III, except at the outflow where a more suitable advective condition is used in conjunction with zero pressure at the upper-right corner of the domain. A limited number of controlled solutions shall be computed for validation purposes using the same method and boundary conditions; see Appendix C 2 for detailed results. We take this opportunity to estimate a posteriori the outflow behavior of the perturbation, that is, the difference between the controlled and the natural solutions. Paying attention to comparing different physical times yet corresponding to the same phase in the shedding cycle (e.g., the peak values of lift), we obtain characteristic magnitudes of the stress of order $|\boldsymbol{\sigma}(\delta p, \delta \boldsymbol{u}) \cdot \boldsymbol{n}| \sim 10^{-5}$ at the outflow. Moreover, we find the latter perturbation to be almost identical to that obtained solving Eqs. (26) — for this purpose only—with an exact stress-free condition. This provides good evidence that the perturbative results are free from numerical effects due to the outflow boundary condition, and supports solving the adjoint problem with the same outflow condition as defined in Sec. III B.

The natural cylinder flow eventually settles down to its time-periodic, vortex-shedding state at the Reynolds number $R e=100$ considered in the remainder of the section. In the limit of infinitesimal control force $\delta \boldsymbol{f}$, the controlled cylinder flow remains periodic but with a different period, ${ }^{1}$ which causes the direct solution $(\delta \boldsymbol{u}, \delta p)$ to beat at a low frequency and prevents performing the sensitivity analysis over a single shedding period. Even so, it remains possible to reduce the problem to periodic direct solutions by scaling the time variable on the period of the solution itself, as has been done by Luchini, Giannetti, and Pralits ${ }^{17,43}$ to analyze the sensitivity of finite-amplitude vortex-shedding in wake past a circular cylinder. Such an approach is well suited to determine the sensitivity of the shedding period, otherwise not an explicit unknown of the problem. Nonetheless, we expect the benefit of doing so would be marginal for our case. Broadly speaking, it would only 


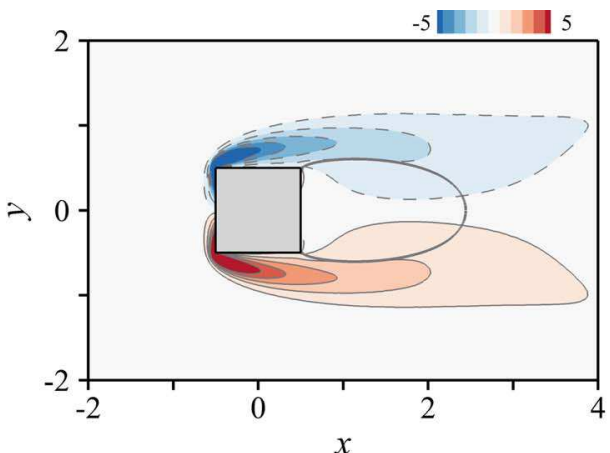

(a)

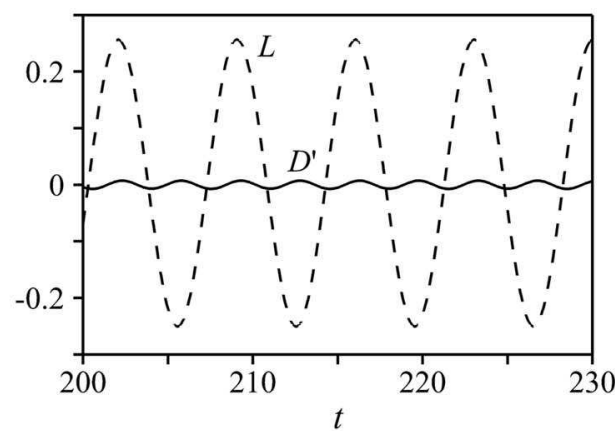

(c)

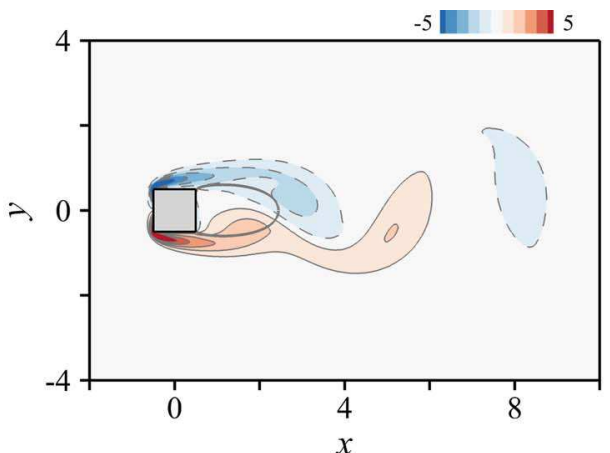

(b)

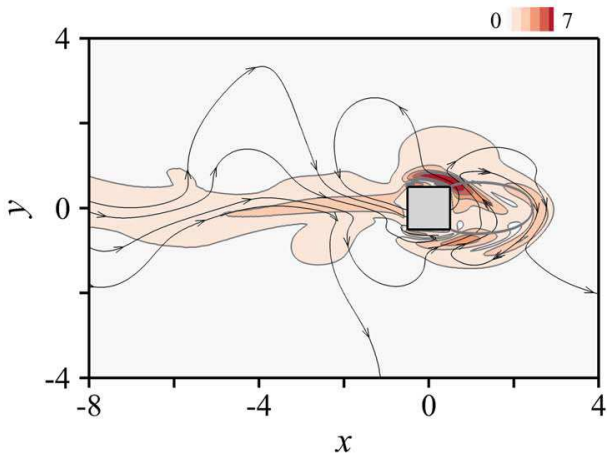

(d)

FIG. 6. Square cylinder flow at $R e=100$. (a) Iso-contours of mean vorticity. The thick gray line is the separatrix of the mean recirculation region. (b) Instantaneous snapshot of the vorticity contours. (c) Time histories of drag (solid line) and lift (dashed line) in the shedding regime. Only the deviation of drag from its mean value is provided to improve readability. (d) Instantaneous snapshot of the mean drag sensitivity. The magnitude of sensitivity is given by the color levels and the orientation of the underlying vector by the superimposed streamlines.

eliminate the need to introduce an initial adjoint condition on behalf of the adjoint solution being periodic as well-a behavior observed in our simulations and previously reported by Wang and $\mathrm{Gao}^{32}$-but computing the sensitivity would require all the same to march backwards in time the adjoint system until a time-periodic state is reached, as noted in the aforementioned studies. For the sake of simplicity, we thus proceed here solving adjoint system (29) from zero initial condition and taking advantage of periodicity to save computational time and resources. We perform first the DNS of the natural cylinder flow starting from a random initial condition, typical time step employed being $\Delta t=0.05$ to achieve convergence of the force coefficients in terms of mean and root mean square (rms) values. The periodic regime is reached at $\tau_{1}=100$, whereafter the solution is marched forward in time, stored to disk at each time-step and averaged on-the-fly over 500 additional time units, up to $\tau_{f}=600$. Then, we initialize the adjoint solution to zero at $\tau_{f}$ and solve the adjoint system using the same time-step, the DNS solution required to achieve discretization of the adjoint advection operator being simply read from disk. The periodic regime is reached at $\tau_{2}=500$, whereafter the adjoint solution is marched backwards in time down to $\tau_{1}$. Finally, we average all sensitivity integrands $\left(\boldsymbol{u}^{\dagger} \mid \boldsymbol{\delta} \boldsymbol{f}\right.$ ) over the same interval $\left(\tau_{1}, \tau_{2}\right)$ to leave out the transient effect of the initial conditions, the corresponding time-span of 400 time units (equivalently about 55 shedding cycles) being suitable to converge meaningful averages even though not an integer multiple of the period.

The contours of mean vorticity presented in Fig. 6(a) closely resemble those of the base solution shown in Fig. 2(a). Namely, two shear layers displaying vorticity of opposite signs form at the upstream stagnation point, remain attached on the top/bottom sides of the cylinder and separate at the trailing edges, as evidenced by the thick gray line representing the mean separating streamline. This is consistent with the observations of Robichaux, Balachandar, and Vanka ${ }^{44}$ who report separation from the leading edges for Reynolds numbers above 120. The recirculation extends 1.94 cylinder 
diameters downstream of the base. The mean drag is $\bar{D}=1.46$. A snapshot of the vorticity contours is presented in Fig. 6(b) to evidence the roll-up of the shear layers and the formation of the large-scale vortices shed periodically in the wake of the cylinder. Typical time histories of drag (solid line) and lift (dashed line) are provided in Fig. 6(c) - actually we report the drag fluctuation $D^{\prime}$ to improve readability because the amplitude of the lift oscillations is about two orders of magnitude larger than that of the drag oscillations ( 0.51 vs. 0.014$)$. The shedding frequency estimated by spectral analysis of the lift signal is $f=0.14$. All numerical values are in good agreement with existing data. ${ }^{35,40,44}$ Finally, a snapshot of the sensitivity is shown in Fig. 6(d). Although the result is only an instantaneous vision of the adjoint topology, it can be inferred that the magnitude of sensitivity is large all around the cylinder with maxima located upstream of the cylinder and closer to the trailing edges, just as in the steady case at $R e=40$. The main difference is that the sensitivity is now almost zero downstream of the recirculation region, even though the magnitude of vorticity remains significant in the shear layer; see Fig. 2(b) for comparison.

\section{Application to open-loop control by a small circular cylinder}

We now use the sensitivity as a systematic path to guide the best positions for placement of a control cylinder in the attempt to reduce the mean drag. We model the presence of this secondary cylinder by the pointwise reacting force

$$
\boldsymbol{\delta} \boldsymbol{f}_{\eta}(x, y)=-\frac{1}{2} \eta D_{\eta}\left(R e_{\eta}\right) \boldsymbol{\xi}\left(x_{\mathrm{c}}, y_{\mathrm{c}}\right) \delta\left(x-x_{\mathrm{c}}, y-y_{\mathrm{c}}\right),
$$

equal and opposite to that acting on a fictitious cylinder of same diameter subjected to a uniform flow at the local, now time-dependent, flow velocity (we recall that $\boldsymbol{\xi}=\|\boldsymbol{u}\| \boldsymbol{u}$ is the signed square velocity vector). We obtain Eq. (31) from classical unsteady fluid force models ${ }^{45}$ in the quasi-static approximation, i.e., overlooking inertia effects and assuming the force acting on the cylinder at each time instant to be identical to the force that would act if the upstream flow at the same instant was a steady one. For consistency, this requires redefining the local Reynolds number from the averaged magnitude of velocity such that $u_{\mathrm{c}}{ }^{2}=\overline{\|\boldsymbol{u}\|^{2}}\left(x_{\mathrm{c}}, y_{\mathrm{c}}\right)$. The approach has the advantage of simplicity since it requires modeling only the dependence on Reynolds number of the drag coefficient for steady flow. It holds as a first approximation in the limit of small control cylinders because the advection time scale in the vicinity of the control cylinder is then much smaller than the vortex-shedding period. This yields large values of the local Keulegan-Carpenter number $K_{C_{\eta}}=u_{\mathrm{c}} / \eta f$ (typical values are of order of several hundreds for $\eta=0.01$ and $R e=100$ ), a regime where inertia effects are small and the drag coefficient is essentially equal to its value for steady flow. Consequently, we approximate the drag coefficient $D_{\eta}$ using the same power law (14) relevant for diameters of the control cylinder up to $\eta \sim 0.2$ at the considered Reynolds number $R e=100$. Note we do not consider using the Oseen approximation in this case, because Eq. (19) yields divergence of the drag coefficient at Reynolds numbers approaching $R e_{\eta} \sim 7.5$.

We map in Fig. 7(a) the variation of the cylinder mean $\operatorname{drag} \overline{\delta D}$ induced by a control cylinder of diameter $\eta=0.1$ at $R e=100$, for which the local Reynolds number is below 10. To each spatial position $\left(x_{\mathrm{c}}, y_{\mathrm{c}}\right)$ of the control cylinder is associated a drag variation computed as

$$
\overline{\delta D}\left(x_{\mathrm{c}}, y_{\mathrm{c}}\right)=\left(\left(\boldsymbol{u}^{\dagger} \mid \boldsymbol{\delta} \boldsymbol{f}_{\eta}\right)\right)=-\frac{1}{2} \eta D_{\eta} \overline{\boldsymbol{u}^{\dagger}\left(x_{\mathrm{c}}, y_{\mathrm{c}}\right) \cdot \boldsymbol{\xi}\left(x_{\mathrm{c}}, y_{\mathrm{c}}\right)} .
$$

The map is almost perfectly symmetric with respect to the centerline, and exhibits both negative and positive variations corresponding respectively to a decrease or an increase of the mean cylinder drag. It indicates that there are three main regions in which the drag is reduced, a large one upstream of the cylinder, another one located on either side of the recirculating streamline, and a last one extending further downstream in the shear layers. A maximum reduction by $20 \%$ (just as in the steady case at $R e=40$ ) is achieved placing the control cylinder in the upstream region. In contrast, drag increases in the early shear regions originating from the leading edges. Fig. 7(b) proposes a 


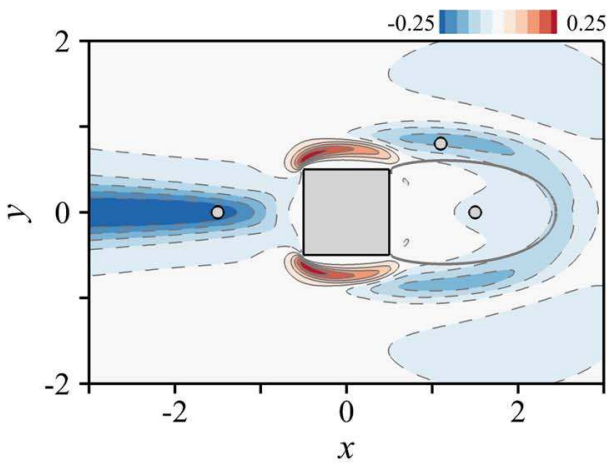

(a)

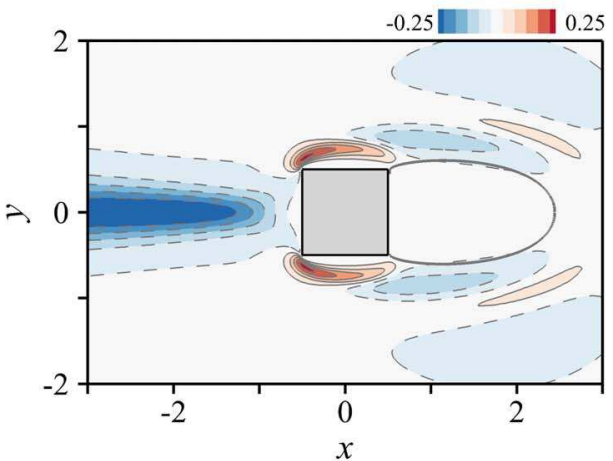

(c)

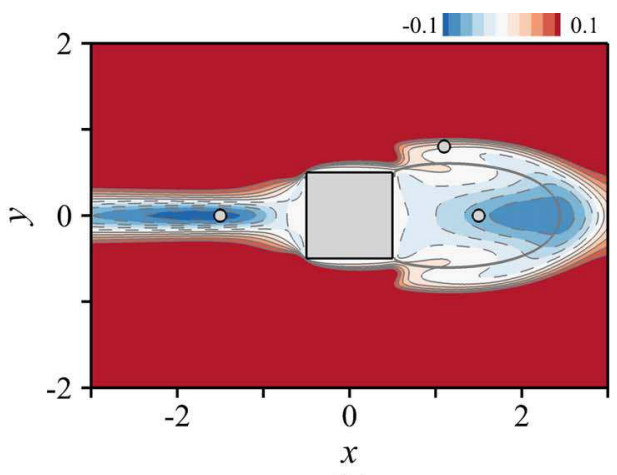

(b)

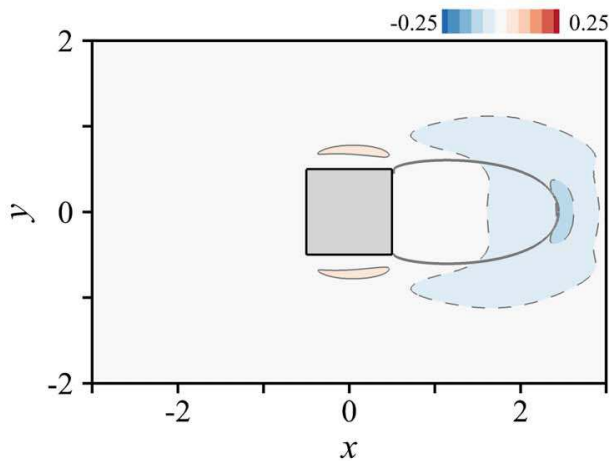

(d)

FIG. 7. (a) and (b) Variation of mean drag induced by a control cylinder of diameter $\eta=0.1$ whose presence is modeled by Eq. (31) $-R e=100$. (a) Drag of the square cylinder. (b) Drag of the two-cylinder system. The various circle symbols mark the positions for which the theoretical predictions are compared to DNS of open-loop control by a small control cylinder in Fig. 8. (c) and (d) Variation of mean drag computed retaining only the (c) mean and (d) fluctuating components of the model force. The map shown in (a) is thus retrieved as the sum of these two maps.

map of the total drag variation

$$
\overline{\delta D}_{\text {tot }}\left(x_{\mathrm{c}}, y_{\mathrm{c}}\right)=-\frac{1}{2} \eta D_{\eta} \overline{\left(\boldsymbol{u}^{\dagger}\left(x_{\mathrm{c}}, y_{\mathrm{c}}\right)-2 \boldsymbol{e}_{\boldsymbol{x}}\right) \cdot \boldsymbol{\xi}\left(x_{\mathrm{c}}, y_{\mathrm{c}}\right)},
$$

representing the variation between the mean drag of the two-cylinder system and that of the natural cylinder flow. It indicates that the total drag is reduced in the upstream region of largest sensitivity with maximum achieved reduction by $11 \%$ (twice as much as in the steady case), but large negative values prevail also in the inner recirculation region, which is because the drag of the main cylinder is reduced and the control cylinder is a source of thrust, both effects adding to one another in Eq. (33).

It is suggested here that the control cylinder acts primarily via the mean component of the force. Indeed, the main regions yielding either a decrease or an increase of the cylinder drag are retrieved with a fairly good agreement retaining only the mean component of the force, while the variation induced by its fluctuation is systematically lower by one order of magnitude; see Figs. 7(c) and 7(d) showing maps of the individual variations

$$
\left(\left(\boldsymbol{u}^{\dagger} \mid \overline{\boldsymbol{\delta} \boldsymbol{f}_{\eta}}\right)\right)=\left(\overline{\boldsymbol{u}^{\dagger}} \mid \overline{\boldsymbol{\delta} \boldsymbol{f}_{\eta}}\right)=-\frac{1}{2} \eta D_{\eta} \overline{\boldsymbol{u}^{\dagger}}\left(x_{\mathrm{c}}, y_{\mathrm{c}}\right) \cdot \overline{\boldsymbol{\xi}}\left(x_{\mathrm{c}}, y_{\mathrm{c}}\right),
$$

and

$$
\left(\left(\boldsymbol{u}^{\dagger} \mid \boldsymbol{\delta} \boldsymbol{f}_{\eta}^{\prime}\right)\right)=\left(\left(\boldsymbol{u}^{\dagger \prime} \mid \boldsymbol{\delta} \boldsymbol{f}_{\eta}^{\prime}\right)\right)=-\frac{1}{2} \eta D_{\eta} \overline{\boldsymbol{u}^{\dagger \prime}\left(x_{\mathrm{c}}, y_{\mathrm{c}}\right) \cdot \boldsymbol{\xi}^{\prime}\left(x_{\mathrm{c}}, y_{\mathrm{c}}\right)}
$$




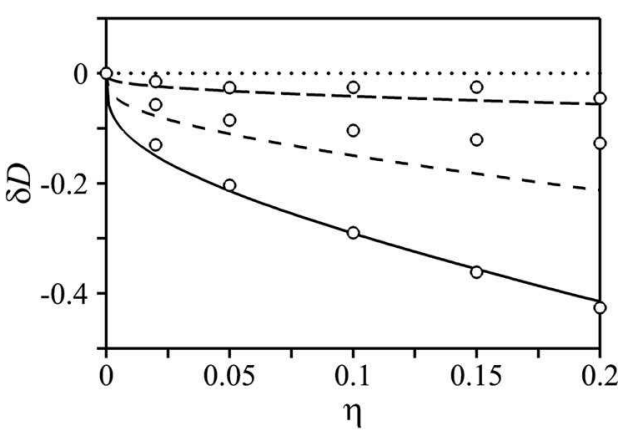

(a)

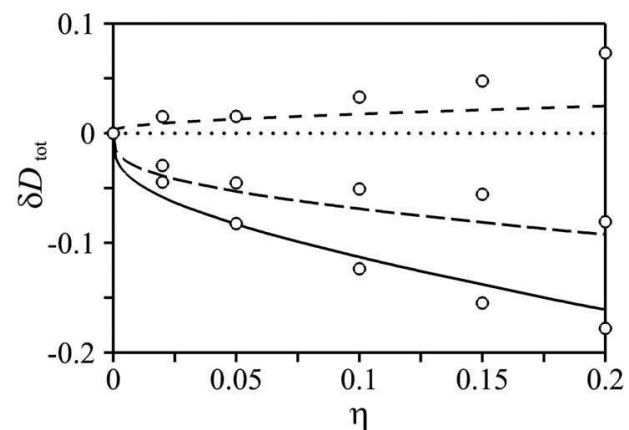

(b)

FIG. 8. Variation of mean drag as a function of the diameter of the control cylinder at $R e=100$. The black lines denote theoretical predictions obtained modeling the presence of the cylinder at positions $\left(x_{\mathrm{c}}, y_{\mathrm{c}}\right)=(-1.5,0)$ (solid line), $\left(x_{\mathrm{c}}, y_{\mathrm{c}}\right)=$ $(1.1,0.8)$ (dashed line), and $\left(x_{\mathrm{c}}, y_{\mathrm{c}}\right)=(1.5,0)$ (long-dashed line) by Eq. (31). The exact values obtained from DNS of flow over the two cylinders are superimposed as the open symbols. (a) Drag of the square cylinder. (b) Drag of the two-cylinder system.

using the same color look-up table. This is not true however in the rear part of the recirculation where the mean force has almost no effect, meaning that the drag reduction observed in Fig. 7(a) is driven by the fluctuating component of the force.

\section{Comparison with mean drag of the two-cylinder system}

The black lines in Fig. 8 stand for the variations $\overline{\delta D}$ and $\overline{\delta D}_{\text {tot }}$ computed from Eqs. (32) and (33) placing the control cylinder at the three positions marked by the symbols in Fig. 7 and varying its diameter up to $\eta=0.2$. The solid line is for the first position $\left(x_{\mathrm{c}}, y_{\mathrm{c}}\right)=(-1.5,0)$ where the control cylinder decreases both the cylinder and the total drag, the dashed line is for the second position $\left(x_{\mathrm{c}}\right.$, $\left.y_{\mathrm{c}}\right)=(1.1,0.8)$ where it decreases the cylinder drag but increases the total drag, and the long-dashed line is for the third position $\left(x_{\mathrm{c}}, y_{\mathrm{c}}\right)=(1.5,0)$ where it decreases again both the cylinder and the total drag. The superimposed symbols are numerical data points of open-loop control by means of a small control cylinder, computed from DNS of the two-cylinder system with 300 points distributed at the surface of the control cylinder. The obtained results follow the theoretical trends and exhibit a satisfactory quantitative agreement. This is especially true at the first position $\left(x_{\mathrm{c}}, y_{\mathrm{c}}\right)=(-1.5,0)$, consistently with the amplitude of the flow oscillations being limited upstream of the main cylinder. For the other two cases, there exist discrepancies at non-small values of $\eta$, which can be ascribed to the fact that the quasi-static assumption becomes questionable, for instance, at $\eta=0.1$ (resp., $\eta=0.2)$, the Keulegan-Carpenter number is of order $K_{C_{\eta}} \sim 50$ (resp., 25) at $\left(x_{\mathrm{c}}, y_{\mathrm{c}}\right)=(1.1,0.8)$ and $K_{C \eta} \sim 20$ (resp., 10) at $\left(x_{\mathrm{c}}, y_{\mathrm{c}}\right)=(1.5,0)$. These values fall into the inertia-drag regime in which inertia effects become significant and the drag coefficient differs from its value for steady flow. The reliability of the model could be improved by fitting individually drag and inertia coefficients from numerical simulations of circular cylinder subjected to an orbital flow in the appropriate ranges of $\left(R e_{\eta}, K_{C \eta}\right)$, but this lies out of the scope of the study, as we believe the present basic modeling already demonstrates the ability of the approach in providing qualitative and fairly quantitative predictions.

\section{E. Effect upon the mean lift and the fluctuating forces}

Only minor modification to the above theoretical framework is required to assess the effect of the control cylinder upon the mean lift, as we show in Appendix C 3 that the related sensitivity function $\nabla_{f} \bar{L}$, deduces from the solution to the adjoint equations (29) with boundary condition at the cylinder surface

$$
\boldsymbol{u}^{\dagger}=2 \boldsymbol{e}_{\boldsymbol{y}} \quad \text { on } \Gamma_{\mathrm{cyl}} .
$$




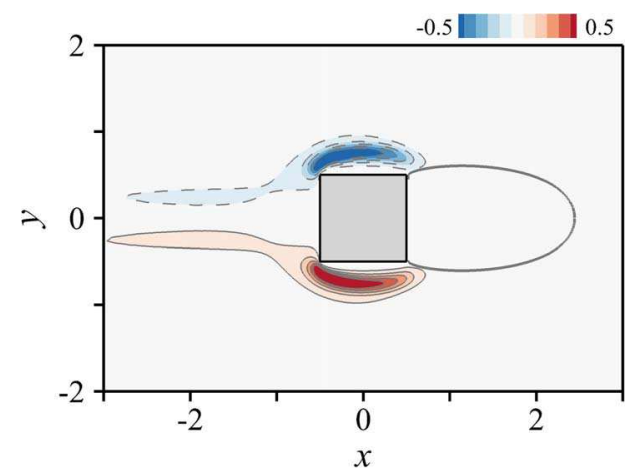

FIG. 9. Variation of mean lift induced by a control cylinder of diameter $\eta=0.1$ whose presence is modeled by Eq. (31) - Re $=100$.

In return, the lift variation computed from the model force (31) and mapped in Fig. 9 indicates that a control cylinder of diameter $\eta=0.1$ increases the mean lift at $R e=100$ if positioned in the shear region originating from the lower leading edge, spreading on the cylinder length over the bottom side and extending further upstream, up to 2-3 diameters of the main cylinder.

It is hardly more complicated to assess the effect upon the fluctuating drag and lift, as measured by their rms values

$$
D_{\mathrm{rms}}^{2}=\frac{1}{\tau} \int_{0}^{\tau} D^{\prime 2} \mathrm{~d} t \quad \text { and } \quad L_{\mathrm{rms}}^{2}=\frac{1}{\tau} \int_{0}^{\tau} L^{\prime 2} \mathrm{~d} t,
$$

as we show in Appendix C 4 that the related sensitivity functions $\nabla_{f} D_{\mathrm{rms}}$ and $\nabla_{f} L_{\mathrm{rms}}$ deduce from the solution to the adjoint equations (29) with boundary condition at the cylinder surface either

$$
\boldsymbol{u}^{\dagger}=2 \frac{D^{\prime}}{D_{\mathrm{rms}}} \boldsymbol{e}_{\boldsymbol{x}} \quad \text { on } \Gamma_{\mathrm{cyl}}
$$

for rms drag, or

$$
\boldsymbol{u}^{\dagger}=2 \frac{L^{\prime}}{L_{\mathrm{rms}}} \boldsymbol{e}_{\boldsymbol{y}} \quad \text { on } \Gamma_{\mathrm{cyl}},
$$

for rms lift. In return, the variation of rms drag mapped in Fig. 10(a) indicates that a control cylinder of diameter $\eta=0.1$ decreases the rms drag at $R e=100$ if positioned upstream of the cylinder, in the inner recirculation region or along its external boundary, i.e., almost exactly the same zones where it reduces the mean drag. The structure of the map is however more complex since it conversely

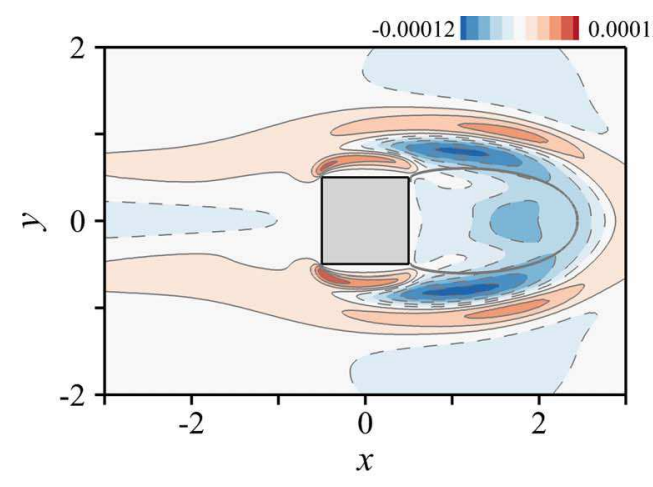

(a)

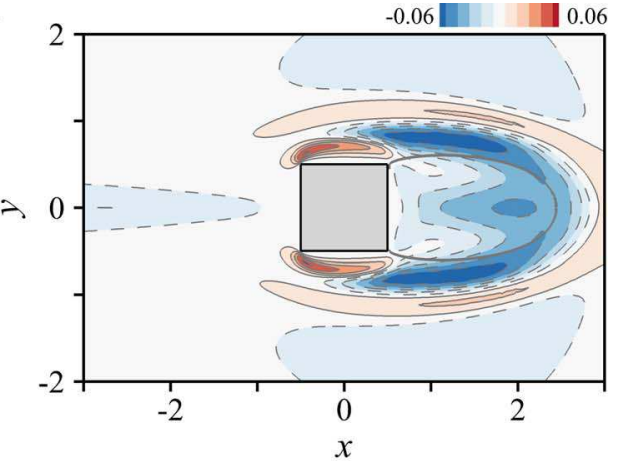

(b)

FIG. 10. Variation of the (a) rms drag and (b) rms lift induced by a control cylinder of diameter $\eta=0.1$ whose presence is modeled by Eq. (31) - $R e=100$. 
increases the rms drag if positioned in the narrow shear region originating from the leading edges now extending upstream, offset from the centerline, as well as in a second large region surrounding the recirculation. Despite some local differences, the map of the rms lift variation shown in Fig. 10(b) is somehow similar. It displays variations larger by two orders of magnitudes, which stems not from a lower sensitivity, rather from the amplitude of the lift oscillations being much larger than its drag counterpart, as has been said in Sec. IV B. Given the rms values of drag and lift $\left(D_{\mathrm{rms}}=0.0051\right.$ and $\left.L_{\mathrm{rms}}=0.18\right)$ the relative variations achieved in both cases are actually comparable.

\section{SIMPLIFIED STEADY SENSITIVITY ANALYSIS IN LAMINAR UNSTEADY FLOW REGIME}

Because drag is linear in the flow variables, the mean drag can be viewed as a steady function of only the mean flow variables

$$
\bar{D}=\frac{2}{\tau} \int_{0}^{\tau} \oint_{\Gamma_{\mathrm{cyl}}}\{\boldsymbol{\sigma}(p, \boldsymbol{u}) \cdot \boldsymbol{n}\} \cdot \boldsymbol{e}_{\boldsymbol{x}} \mathrm{d} l \mathrm{~d} t=2 \oint_{\Gamma_{\mathrm{cyl}}}\{\boldsymbol{\sigma}(\bar{p}, \overline{\boldsymbol{u}}) \cdot \boldsymbol{n}\} \cdot \boldsymbol{e}_{\boldsymbol{x}} \mathrm{d} l .
$$

Building on this, we seek here to determine the extent to which correct levels of sensitivity-and thereby relevant control predictions - can be obtained from knowledge of only the mean cylinder flow. We believe the answer can provide some insight into how reasonable comparisons can be made between purely theoretical results and more practical situations, as the history of time and space-accurate solutions required to perform the exact sensitivity analysis is likely not to be available from standard numerical simulations or experimental measurements.

\section{A. Theoretical framework}

The present approach is closely related to existing studies considering the mean flow an admissible solution for linear stability and sensitivity analyses. Barkley ${ }^{46}$ has especially highlighted that analyzing the mean flow in that way assumes the Reynolds stresses of the fluctuating velocity to be unperturbed at linear order, as can be seen from the classical mean flow equations

$$
\begin{aligned}
& \nabla \cdot \overline{\boldsymbol{u}}=\mathbf{0}, \quad \nabla \overline{\boldsymbol{u}} \cdot \overline{\boldsymbol{u}}-\nabla \cdot \boldsymbol{\sigma}(\bar{p}, \overline{\boldsymbol{u}})=\overline{\boldsymbol{\psi}\left(\boldsymbol{u}^{\prime}\right)} \quad \text { in } \Omega, \\
& \overline{\boldsymbol{u}}=\mathbf{0} \quad \text { on } \Gamma_{\mathrm{cyl}},
\end{aligned}
$$

defining $(\overline{\boldsymbol{u}}, \bar{p})$ as a solution to the steady Navier-Stokes equations forced by the Reynolds stresses $\overline{\boldsymbol{\psi}\left(\boldsymbol{u}^{\prime}\right)}=-\overline{\nabla \boldsymbol{u}^{\prime} \cdot \boldsymbol{u}^{\prime}}$. The relationship between the base flow (as defined by Eq. (5), thus solution to Eq. (41) with zero right-hand side) and the mean flow in cylinder wakes and related flows has been discussed extensively, the general picture being that a mean flow correction, through coupling with the fluctuating motion and formation of Reynolds stresses, is the mechanism for nonlinear saturation of the oscillatory instability. ${ }^{47-50}$ The effect of this mean flow correction is illustrated in Fig. 11 comparing the contours of the mean vorticity obtained by DNS at $R e=100$-hence reproduced from Fig. 6(a)—and those of the base solution computed at the same Reynolds number using the Newton-Raphson method. Both solutions are similar in broad strokes but the symmetric recirculation of the base solution extends up to 8 diameters downstream of the cylinder base, which is 4 times as much as its mean counterpart.

If we do assume the Reynolds stresses unperturbed (i.e., if we overlook the nonlinear coupling between the mean flow perturbation induced by the control and its fluctuation, as further explained in Appendix A), the steady equations governing the mean flow perturbation become

$$
\begin{array}{rr}
\nabla \cdot \overline{\boldsymbol{\delta} \boldsymbol{u}_{0}}=\mathbf{0}, \quad \nabla \overline{\boldsymbol{\delta} \boldsymbol{u}_{0}} \cdot \overline{\boldsymbol{u}}+\nabla \overline{\boldsymbol{u}} \cdot \overline{\boldsymbol{\delta} \boldsymbol{u}_{0}}-\nabla \cdot \boldsymbol{\sigma}\left(\overline{\delta p_{0}}, \overline{\boldsymbol{\delta} \boldsymbol{u}_{0}}\right)=\overline{\boldsymbol{\delta} \boldsymbol{f}} & \text { in } \Omega, \\
\overline{\boldsymbol{\delta} \boldsymbol{u}_{0}}=\mathbf{0} & \text { on } \Gamma_{\mathrm{cyl}},
\end{array}
$$

the subscripts " 0 " being used on purpose to clarify that the solution to Eq. (42) is only an approximation to the exact mean flow perturbation. The key point is that the above direct system is formally 


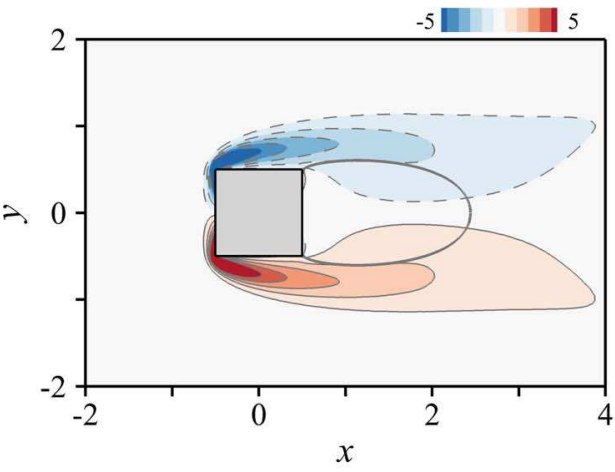

(a)

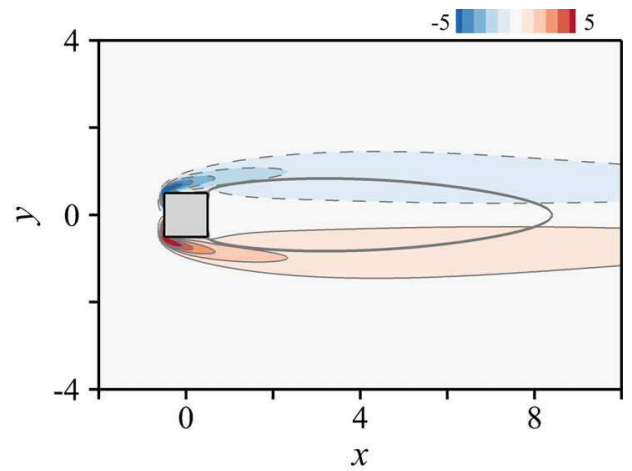

(b)

FIG. 11. Iso-contours of (a) mean and (b) base vorticity at $R e=100$.

identical to that (7) derived in steady flow regime, the only differences being that (i) linearization is performed about the mean flow quantities and (ii) only the mean component of the control force shows up as an additional right-hand side. In return, we can thus introduce the solution $\left(\overline{\boldsymbol{u}_{0}^{\dagger}}, \overline{p_{0}^{\dagger}}\right)$ to the steady adjoint system

$$
\begin{aligned}
& \boldsymbol{\nabla} \cdot \overline{\boldsymbol{u}_{0}^{\dagger}}=\mathbf{0}, \quad-\nabla \overline{\boldsymbol{u}_{0}^{\dagger}} \cdot \overline{\boldsymbol{u}}+\nabla \overline{\boldsymbol{u}} \cdot \overline{\boldsymbol{u}_{0}^{\dagger}}-\nabla \cdot \boldsymbol{\sigma}\left(-\overline{p_{0}^{\dagger}}, \overline{\boldsymbol{u}_{0}^{\dagger}}\right)=\mathbf{0} \quad \text { in } \Omega, \\
& \overline{\boldsymbol{u}_{0}^{\dagger}}=2 \boldsymbol{e}_{\boldsymbol{x}} \text { on } \Gamma_{\text {cyl }},
\end{aligned}
$$

and infer all quantities to be such that

$$
\int_{\Omega} \overline{\boldsymbol{u}_{0}^{\dagger}} \cdot \overline{\boldsymbol{\delta} \boldsymbol{f}} \mathrm{d} x \mathrm{~d} y=\oint_{\Gamma_{\mathrm{cy} 1}}\left\{\boldsymbol{\sigma}\left(\overline{\delta p_{0}}, \overline{\boldsymbol{\delta} \boldsymbol{u}_{0}}\right) \cdot \boldsymbol{n}\right\} \cdot \overline{\boldsymbol{u}_{0}^{\dagger}} \mathrm{d} l=\overline{\delta D_{0}},
$$

where $\overline{\delta D_{0}}$ physically represents the mean drag variation computed without coupling from the Reynolds stresses, used as a first approximation to the exact variation $\overline{\delta D}$.

The assumption is somehow straight, for instance, Eq. (44) readily expresses that it amounts to purely missing the effect of the fluctuating force. Nevertheless, we believe the approach offers an interesting compromise between giving consistent predictions of acceptable quality within limited computational effort. On the one hand, assuming unperturbed Reynolds stresses has proved fruitful in retrieving complex spatio-temporal features of the shedding regime (e.g., shedding frequency, characteristic wavelength of the Kármán vortex street, sensitivity of the shedding activity) from linear analysis of laminar and turbulent mean wakes ${ }^{26,27,46,50-55}$ and in relating near-wall streaks involved in the production of channel flow turbulence to linear perturbations of the turbulent mean flow, ${ }^{56,57}$ which gives hope that it can also predict reasonably well the mean drag variations induced by a small control cylinder. We expect in particular the error made overlooking the fluctuating component of the reacting force to be limited because the control cylinder has been said to act primarily via the mean component; see Sec. IV C. On the other hand, the related mean drag variation is obtained solving a single steady adjoint problem with only requirement to be able to compute accurately the mean flow.

\section{B. Application to control by a small circular cylinder}

The method is applied here to the unsteady flow at $R e=100$ considered in Sec. IV. The line of thought being to derive control predictions from knowledge of only the mean cylinder flow, it makes sense to replace the mean component of the reacting force (31) reading

$$
\overline{\boldsymbol{\delta} \boldsymbol{f}_{\eta}}(x, y)=-\frac{1}{2} \eta D_{\eta}\left(R e_{\eta}\right) \overline{\boldsymbol{\xi}}\left(x_{\mathrm{c}}, y_{\mathrm{c}}\right) \delta\left(x-x_{\mathrm{c}}, y-y_{\mathrm{c}}\right),
$$




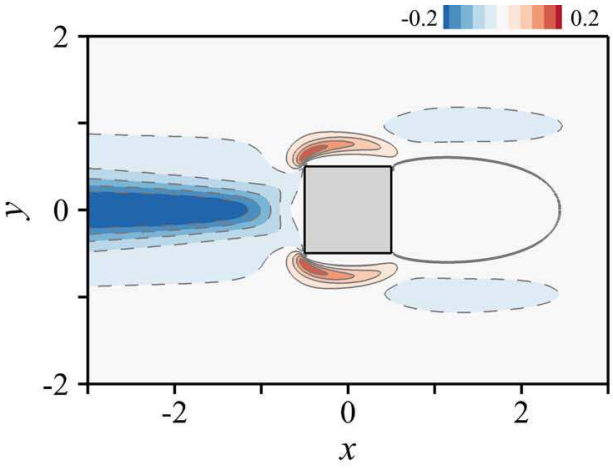

(a)

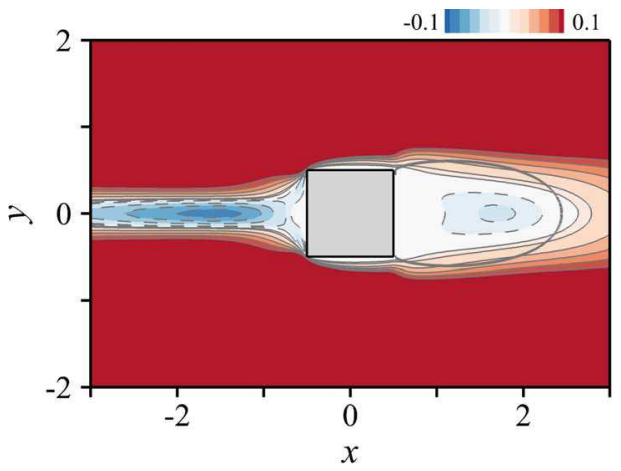

(b)

FIG. 12. Variation of mean drag induced by a control cylinder of diameter $\eta=0.1$ whose presence is modeled by Eq. (46), computed without coupling from the Reynolds stresses - $R e=100$. (a) Drag of the square cylinder. (b) Drag of the two-cylinder system.

and involving the (unknown) averaged signed square velocity vector $\overline{\boldsymbol{\xi}}=\overline{\|\boldsymbol{u}\| \boldsymbol{u}}$, by its approximation

$$
\widetilde{\boldsymbol{\delta}}_{\eta}(x, y)=-\frac{1}{2} \eta D_{\eta}\left(R e_{\eta}\right) \widetilde{\boldsymbol{\xi}}\left(x_{\mathrm{c}}, y_{\mathrm{c}}\right) \delta\left(x-x_{\mathrm{c}}, y-y_{\mathrm{c}}\right),
$$

involving only the signed square averaged velocity vector $\widetilde{\boldsymbol{\xi}}=\|\overline{\boldsymbol{u}}\| \overline{\boldsymbol{u}}$. Provided the local Reynolds number is redefined from the magnitude of averaged velocity $u_{\mathrm{c}}=\|\overline{\boldsymbol{u}}\|\left(x_{\mathrm{c}}, y_{\mathrm{c}}\right)$, this is equivalent to modeling the presence of the cylinder by a reacting force equal and opposite to the force acting on a fictitious cylinder of same diameter subjected to a uniform flow at the local, mean velocity as has been done by Meliga et al. ${ }^{26}$ Both expressions (45) and (46) differ because the average of the square is not the square of the average, but we expect the approximation to be quite accurate in so far as the fluctuating motion has been shown to produce only second-order corrections to the mean drag sensitivity; see Figs. 7 (c) and 7(d). The approximated mean drag variations $\overline{\delta D_{0}}$ and $\overline{\delta D_{0}}$ tot deduce as

$$
\overline{\delta D_{0}}\left(x_{\mathrm{c}}, y_{\mathrm{c}}\right)=-\frac{1}{2} \eta D_{\eta} \overline{\boldsymbol{u}_{0}^{\dagger}}\left(x_{\mathrm{c}}, y_{\mathrm{c}}\right) \cdot \tilde{\boldsymbol{\xi}}\left(x_{\mathrm{c}}, y_{\mathrm{c}}\right),
$$

and

$$
\left.\overline{\delta D_{0 \text { tot }}}\left(x_{\mathrm{c}}, y_{\mathrm{c}}\right)=-\frac{1}{2} \eta D_{\eta} \overline{\left(\boldsymbol{u}_{0}^{\dagger}\right.}\left(x_{\mathrm{c}}, y_{\mathrm{c}}\right)-2 \boldsymbol{e}_{\boldsymbol{x}}\right) \cdot \tilde{\boldsymbol{\xi}}\left(x_{\mathrm{c}}, y_{\mathrm{c}}\right)
$$

whose maps computed for $\eta=0.1$ are presented in Fig. 12. Comparison with the exact maps documented in Fig. 7 provides clear evidence that the simplified framework does carry valuable information in view of guiding efficient control strategy, since the main regions yielding either a decrease or an increase of drag are retrieved satisfactorily at leading order. Namely, we find a maximum drag reduction by $20 \%$ in the large region extending upstream of the main cylinder, a reduction of lesser importance in a secondary region located on either side of the recirculating streamline, but conversely an increase in the early shear regions originating from the leading edges, all effects being fully consistent with the results of Fig. 7. The approach however fails to predict the drag reduction occurring in the recirculation region, which is not too surprising since the control has been said to act mainly via the fluctuating component of the force in this region; see Fig. 7(c). In return, the reduction of total drag observed in Fig. 12(b) is due to the control cylinder acting as a source of thrust, as in the steady case at $R e=40$. It fails also-however to a lesser extent-in predicting quantitatively the magnitude of the drag reduction in the secondary region surrounding the recirculation, found here to be much narrower. This time, the reason is that the control acts via the mean component of the force, but mainly through coupling between the mean flow perturbation and its fluctuation; see the discrepancy with Fig. 7(c) in this region.

We also assess the effect of the control cylinder upon the mean lift variation, computed with the same degree of approximation from the solution to the steady adjoint equations (43) with boundary 


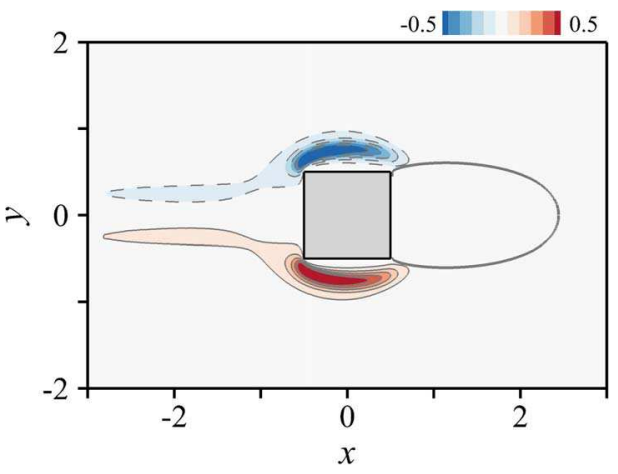

FIG. 13. Variation of mean lift induced by a control cylinder of diameter $\eta=0.1$ whose presence is modeled by Eq. (46), computed without coupling from the Reynolds stresses - $R e=100$.

condition at the cylinder surface

$$
\overline{\boldsymbol{u}_{0}^{\dagger}}=2 \boldsymbol{e}_{\boldsymbol{y}} \quad \text { on } \Gamma_{\mathrm{cyl}} .
$$

The relevance of the approach is even more potent in this case since the map obtained from the model force (46) and presented in Fig. 13 is almost identical to the exact map documented in Fig. 9. This is because lift is significantly altered only upstream of the main cylinder and in the separating shear layer, i.e., precisely the flow regions where the assumptions underlying the simplified framework are expected to hold best on the basis of the above.

Of course, the scope of such simplified analysis is narrower than that of its exact counterpart performed in Sec. IV, for instance, it is impossible to assess the effect of the control cylinder upon the rms drag and lift. While this is consistent with intuition (in the sense that not even the rms of the natural cylinder flow can be obtained from only the mean flow solution), the explanation is best understood from Appendix A where we show that the simplified mean-flow-based steady adjoint system is obtained rigorously decomposing all adjoint quantities into their mean and fluctuating components, substituting in the exact, unsteady adjoint system (29), averaging in time and overlooking the adjoint fluctuating terms. For the rms problem, this yields homogeneous equations and homogeneous conditions at the cylinder surface on behalf of the conditions (38) and (39) being purely fluctuating ones, hence making the adjoint solution and the computable rms variations be trivially zero.

\section{TOWARDS TURBULENT FLOW REGIME}

To the best of the authors' knowledge, it remains an open question whether a meaningful unsteady adjoint solution can be computed in high-Reynolds-number flows exhibiting chaotic features such as sensitivity with respect to initial conditions, as it is generally acknowledged that any method relying on a linearization of the unsteady Navier-Stokes equations will then yield exponentially diverging solutions if the length of the adjoint simulation exceeds the predictability time scale. ${ }^{58,59}$ Providing an answer to this sensitive issue lies out of the scope of the present study, and we will only mention that such blow up of the adjoint solution, together with useless astronomically large magnitudes of sensitivity, have been reported in three-dimensional (3D) flow past a circular cylinder at a Reynolds number as low as $R e=500,{ }^{32}$ in $2 \mathrm{D}$ turbulent wakes at Reynolds numbers of order $10000^{60,61}$ but (quite surprisingly) not in 3D turbulent wakes at about the same Reynolds numbers. ${ }^{30,31}$ Our objective in this final section is to demonstrate that, even so, valuable information regarding the most sensitive regions of such complex flows can be gained from the simplified approach introduced in Sec. V by virtue of its robustness and ease of implementation (we recall that the only prerequisite is that the mean solution must be accurately computable by any appropriate technique, whereupon a single steady adjoint system is solved).

For that purpose, we push the development of the method in the frame of unsteady Reynoldsaveraged Navier-Stokes (RANS) modeling, that is, large-scales are resolved by time-integration 
while small-scales are modeled to provide closure for the averaged Reynolds stresses. Our focus is on the widely benchmarked Reynolds number $R e=22000$ for which the ability of RANS methods in predicting the basic mean flow features and turbulence statistics is assessed in Refs. 62 and 63. Good agreement is evidenced with regards to existing experimental and numerical data, which can be ascribed to the fact that unlike inherently unsteady turbulent flows, there is a clear separation between the large-scale vortices shed in the wake and the small-scale Kelvin-Helmholtzlike instability developing in the shear layers and further yielding the production of turbulence by selective amplification of the background noise. We treat here the flow as being $2 \mathrm{D}$, which we believe constitutes a reasonable approximation prior to attempting to deal with the additional complexity inherent to fully 3D flows in future work.

We use the standard form of the one-equation Spalart-Allmaras turbulence model,${ }^{64}$ therefore the flow motion is described by the velocity $\boldsymbol{u}=(u, v)$, the pressure $p$ and an additional working variable $\tilde{v}$ physically related to the eddy viscosity. The mean flow computation relies on the RANS capability of the OpenFOAM open-source code ${ }^{65}$ We use finite differences for spatial discretization with second-order upwind schemes for the divergence term and second-order centered schemes for the gradients and the laplacian terms. Time discretization relies on the second order Crank-Nicholson scheme. All linear systems are solved using the implemented predictor-corrector-based Pressure Implicit Splitting of Operators (PISO) algorithm. ${ }^{66}$ The dimensions of the computational domain are those $x_{-\infty}=-5, x_{\infty}=15.5$, and $y_{\infty}=7$ recommended by Rodi. ${ }^{62} \mathrm{~A}$ unit stream-wise velocity is imposed at the inflow, where turbulence is forced assuming a ratio of eddy to kinematic viscosity of 0.1. Symmetric conditions are applied at the transverse boundaries, and advective conditions at the outflow. Our reference simulation is for a mesh built with $1185 \times 649$ points $(2894784$ degrees of freedom) distributed with strong clustering close to the walls to capture the near-wall turbulent regions. Typical time step employed is $\Delta t=0.001$. The flow settles down to a fully developed vortex-shedding regime after about 100 time units, whereafter the solution is advanced in time and averaged on-the-fly over 250 additional time units (about 35 shedding cycles). The mean flow is subsequently interpolated on a triangulation of the same domain made of 605357 triangle (3030 043 degrees of freedom), that is, for each degree of freedom in this new grid, we identify the three nearest neighbors belonging to the finite-difference grid and compute all relevant quantities from barycentric interpolation. Finally, we solve the adjoint system using the finite-element RANS solver presented in details in Meliga et al. ${ }^{26}$ and related supplementary material, ${ }^{67}$ which relies on a continuous formulation of the adjoint Spalart-Allmaras equations, including a specific equation for the Lagrange multiplier of the working variable.

The mean vorticity contours are presented in Fig. 14. The mean separating streamline shown as the thick gray line delimits a recirculation region extending 0.60 cylinder diameters downstream of the base. Furthermore, it indicates that the flow now separates from the leading edges, hence involving secondary recirculation regions spreading on the cylinder length over the top/bottom sides. The mean drag is $\bar{D}=2.19$, with rms drag and lift of $D_{\mathrm{rms}}=0.14$ and $L_{\mathrm{rms}}=1.55$, respectively. The shedding frequency estimated by spectral analysis of the lift signal is $f=0.14$. All numerical

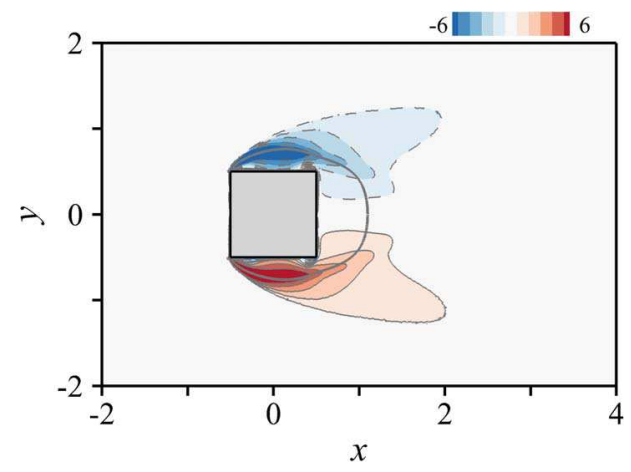

FIG. 14. Iso-contours of mean vorticity at $R e=22000$ obtained by unsteady RANS simulation. 


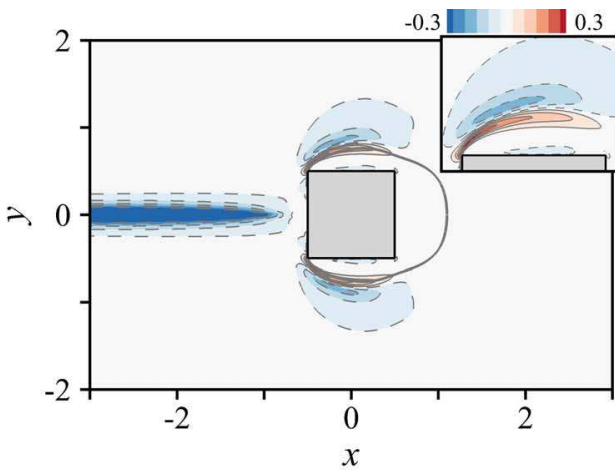

(a)

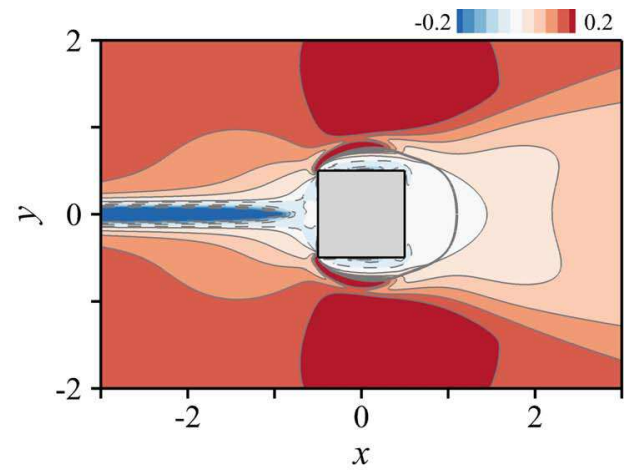

(b)

FIG. 15. Variation of mean drag induced by a control cylinder of diameter $\eta=0.1$ at $R e=22000$, computed in the frame of RANS modeling without coupling from the Reynolds stresses. (a) Drag of the square cylinder. (b) Drag of the two-cylinder system.

values compare well with 2D RANS numerical data available from the literature. ${ }^{63,68,69}$ They are also in reasonable agreement with 3D data from experiments and span-wise averaged large-eddy simulations (LES) ${ }^{69-73}$ which suggests the 2D flow assumption holds true as a first approximation. The main discrepancy lies in the recirculation length, currently underestimated with respect to the reference experimental value 0.88 documented by Lyn et al. ${ }^{70}$ This is not too surprising in so far as the recirculation length is known to be extremely sensitive to the choice of a turbulence model for this flow case, ${ }^{69}$ which may be due to the fact that the inflow is essentially laminar and transition takes place in the separated shear layers developing on either side of the cylinder. This is not taken into account in our simulation since the standard Spalart-Allmaras model assumes fully turbulent behavior. Another explanation is related to the fact that we force the motion to be $2 \mathrm{D}$ and thereby miss intrinsically $3 \mathrm{D}$ effects such as oblique vortex shedding and vortex dislocations.

Computing all drag variations from Eqs. (47) and (48) for $\eta=0.1$ and $R e=22000$ requires to approximate the dependence on Reynolds number of the drag coefficient $D_{\eta}$ up to $R e_{\eta} \sim 2000$. To do so, we use an improved interpolation with larger domain of validity matching our power law (14) to those proposed by Henderson ${ }^{74}$ (steady asymptotic drag if the local Reynolds number is below the critical value $R e_{\eta_{\mathrm{c}}} \sim 46$ for the onset of flow unsteadiness in the wake of the control cylinder and mean drag otherwise), the crossover value being at $R e_{\eta} \sim 19$. The map obtained doing so is presented in Fig. 15(a). It indicates that there are three main regions in which the cylinder drag is reduced, a large one upstream of the cylinder, and two narrow shear regions originating from the leading edges and extending further downstream on either side of the cylinder. The maximum achieved reduction is by $45 \%$ placing the control cylinder in the upstream region, hence suggesting improved controllability with respect to the laminar regime. This is consistent with the experimental results of Igarashi ${ }^{9}$ who reports variations by $30 \%$ positioning the same control cylinder upstream of the main cylinder at a slightly different Reynolds number $R e=32000$. Moving away from the cylinder surface in the cross-wise direction, drag is reduced in a very narrow strip spreading on the cylinder length. It is subsequently increased in a second strip following closely the recirculating streamline, but reduced again in a third strip extending along the outer boundary of the recirculation. Further away, the effect of the control cylinder decays rapidly. A similar alternance of strips-best seen from the close-up in the upper-right corner of Fig. 15(a)—is documented in the experimental results of Sakamoto et al. ${ }^{7}$ despite some differences in the control setting $(\eta=0.07, R e=42000)$. It is uneasy to compare further in the absence of information regarding the spatial resolution of the experimental map, which directly impacts on the achievable level of details. Suffice it to say here that there exists also local discrepancies - for instance we do not retrieve the slight increase of drag observed experimentally in a fourth strip peripheral to the other three-without it being possible to identify a specific cause among the sensitivity analysis possibly overlooking nonlinear mechanisms (owing either to coupling from the Reynolds stresses or to the non-smallness of the control cylinder) 


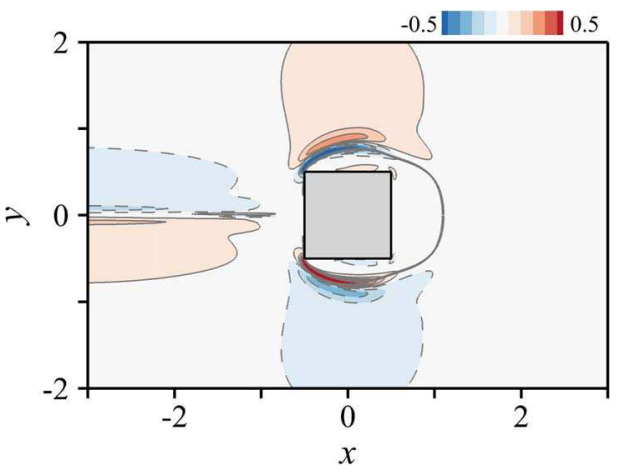

FIG. 16. Variation of mean lift induced by a control cylinder of diameter $\eta=0.1$ at $R e=22000$, computed in the frame of RANS modeling without coupling from the Reynolds stresses.

or the turbulence model possibly lacking accuracy in this specific flow region. Nevertheless, we believe the present results give confidence that our simplified framework can also provide relevant information regarding the most sensitive regions of complex turbulent flows. The map of the total drag variation $\delta D_{0}$ tot computed from Eq. (48) is shown in Fig. 15(b) for the sake of completeness. It indicates that there are essentially two regions left in which the total drag is reduced, namely the region of largest sensitivity located upstream of the cylinder and the vicinity of the top/bottom sides. Note the total drag is slightly increased in the recirculation, which is because the thrust induced by the control cylinder is not sufficient to compensate for the small positive values of $\delta \overline{D_{0}}$ prevailing in this flow region (both effects being barely visible in Fig. 15).

Finally, the map of lift variation obtained for $\eta=0.1$ and $R e=22000$ shown in Fig. 16 indicates that the control cylinder increases the mean lift if positioned not only in the shear region originating from the lower leading edge (as reported herein at $R e=40$ and $R e=100$ ) but also in the outer shear region originating from the upper leading edge, the existence of this second region of interest being consistent with the finding of Sakamoto et $_{\text {al. }}{ }^{7}$

\section{CONCLUSION}

The drag reduction problem in laminar and turbulent flow past a square cylinder is revisited here in the frame of theoretical sensitivity analysis. Namely, we use gradients obtained with the adjoint method to compute the drag variation induced by a body force without calculating the actually controlled states. We then apply the method as a systematic guideline to insert a small secondary circular cylinder, whose presence in the flow is modeled by a reacting force localized at the same location where the control cylinder is placed, equal and opposite to the anticipated drag.

In laminar steady flow regime, we compute the sensitivity of the steady asymptotic drag solving a steady adjoint problem from knowledge of the base solution. At $R e=40$, we find a control cylinder of diameter $\eta=0.1$ to reduce the cylinder drag if positioned in an upstream region extending over several tens of diameters of the main cylinder, or downstream along the outer boundary of the recirculation region. The maximum reduction is by $20 \%$, but the control cylinder itself being a source of drag, it reduces the total drag-i.e., the drag of the two-cylinder system-only in the upstream region, and the maximum reduction drops to 5\%. In laminar unsteady flow regime, we compute the sensitivity of the mean drag integrating backwards in time an unsteady adjoint problem from knowledge of the entire history of the time-dependent cylinder flow. At $R e=100$, we find the same control cylinder to reduce drag if positioned either upstream of the main cylinder, or on either side of the recirculating streamline, or further downstream in the shear layers. The maximum reduction is by $20 \%$, just as in the steady case at $R e=40$. The control cylinder reduces the total drag in the upstream region and in the recirculation region, the maximum reduction being by $11 \%$, twice as much as in the steady case. In both cases, the exact variations computed from numerical 
simulations of the two-cylinder system exhibit very good agreement with the theoretical predictions, hence providing good evidence of relevance.

We also introduce a simplified framework for unsteady flow regime consisting of overlooking the nonlinear coupling between the mean flow perturbation induced by the control and its fluctuation, in which case the sensitivity can be computed at a low computational cost from a steady adjoint problem requiring knowledge of only the mean solution. As is explained herein, the approach is relevant on behalf of the effect of the control cylinder being driven primarily by the mean component of the force it exerts on the flow. The related sensitivity is shown to carry valuable information in view of guiding efficient control strategy in so far as the main regions yielding either a decrease or an increase of drag are retrieved satisfactorily in the laminar unsteady case at $R e=100$. The method is also applied in turbulent flow regime at Reynolds number $R e=22000$, where theoretical predictions obtained in the frame of 2D, unsteady Reynolds-averaged Navier-Stokes modeling upstream of the main cylinder and in the separating shear layers are found consistent with experimental data reported in the literature at comparable control settings. This gives hope the method can carry over at high Reynolds numbers, although further work is needed to confirm the present conclusions.

\section{APPENDIX A: LINK BETWEEN THE SIMPLIFIED AND EXACT UNSTEADY SENSITIVITY FRAMEWORKS}

In this appendix, we discuss the unsteady adjoint system (29) introduced in Sec. IV in the frame of the classical mean/fluctuating decomposition, with the idea to shed some new light on the assumptions underlying the derivation of the simplified sensitivity framework used in Secs. V and VI.

Decomposing all quantities into their mean and fluctuating components, substituting in (29) and averaging in time first yields the steady equations governing the mean adjoint solution $\left(\overline{\boldsymbol{u}^{\dagger}}, \overline{p^{\dagger}}\right)$

$$
\begin{array}{r}
\boldsymbol{\nabla} \cdot \overline{\boldsymbol{u}^{\dagger}}=\mathbf{0}, \quad-\nabla \overline{\boldsymbol{u}^{\dagger}} \cdot \overline{\boldsymbol{u}}+\nabla \overline{\boldsymbol{u}} \cdot \overline{\boldsymbol{u}^{\dagger}}-\nabla \cdot \sigma\left(-\overline{p^{\dagger}}, \overline{\boldsymbol{u}^{\dagger}}\right)=-\underbrace{\overline{\nabla \boldsymbol{u}^{\prime \mathrm{T}} \cdot \boldsymbol{u}^{\dagger \prime}}}_{(\mathrm{b} 1)}+\underbrace{\overline{\nabla \boldsymbol{u}^{\dagger \prime} \cdot \boldsymbol{u}^{\prime}}}_{(\mathrm{c} 1)} \text { in } \Omega, \\
\overline{\boldsymbol{u}^{\dagger}}=2 \boldsymbol{e}_{\boldsymbol{x}} \quad \text { on } \Gamma_{\mathrm{cyl}} .
\end{array}
$$

Subtracting (A1) from (29) then yields the unsteady equations governing its fluctuation $\left(\boldsymbol{u}^{\dagger \prime}, \boldsymbol{p}^{\dagger \prime}\right)$

$$
\begin{aligned}
& \nabla \cdot \boldsymbol{u}^{\dagger \prime}=\mathbf{0}, \quad-\partial_{t} \boldsymbol{u}^{\dagger \prime}-\nabla \boldsymbol{u}^{\dagger \prime} \cdot \boldsymbol{u}^{\prime}+\nabla \boldsymbol{u}^{\prime \mathrm{T}} \cdot \boldsymbol{u}^{\dagger \prime}-\nabla \cdot \sigma\left(-p^{\dagger \prime}, \boldsymbol{u}^{\dagger \prime}\right)= \\
& \overline{\nabla \boldsymbol{u}^{\prime \mathrm{T}} \cdot \boldsymbol{u}^{\dagger \prime}}-\overline{\nabla \boldsymbol{u}^{\dagger \prime} \cdot \boldsymbol{u}^{\prime}} \\
& +\underbrace{\nabla \overline{\boldsymbol{u}^{\dagger}} \cdot \boldsymbol{u}^{\prime}-\nabla \boldsymbol{u}^{\prime \mathrm{T}} \cdot \overline{\boldsymbol{u}^{\dagger}}}_{\text {(a) }}+\underbrace{\nabla \boldsymbol{u}^{\dagger \prime} \cdot \overline{\boldsymbol{u}}}_{\text {(b2) }}-\underbrace{\nabla \overline{\boldsymbol{u}}^{\mathrm{T}} \cdot \boldsymbol{u}^{\dagger \prime}}_{\text {(c2) }} \text { in } \Omega, \\
& \boldsymbol{u}^{\dagger \prime}=\mathbf{0} \quad \text { on } \Gamma_{\text {cyl }} \text {, } \\
& \boldsymbol{u}^{\dagger \prime}=\mathbf{0} \quad \text { at } t=\tau \text {. }
\end{aligned}
$$

Both systems ${ }^{75}$ are coupled through a number of source terms whose origin is best discussed from those coupling the nonlinear equations governing the mean cylinder flow and its fluctuation, obtained applying the exact same procedure to the Navier-Stokes equations. On the one side, we retrieve the steady mean flow equations (41) defining $(\overline{\boldsymbol{u}}, \bar{p})$ as a solution to the steady Navier-Stokes equations forced by the Reynolds stresses. On the other side, we obtain unsteady equations

$$
\begin{aligned}
& \boldsymbol{\nabla} \cdot \boldsymbol{u}^{\prime}=\mathbf{0}, \quad \partial_{t} \boldsymbol{u}^{\prime}+\nabla \boldsymbol{u}^{\prime} \cdot \boldsymbol{u}^{\prime}-\nabla \cdot \boldsymbol{\sigma}\left(p^{\prime}, \boldsymbol{u}^{\prime}\right)=-\overline{\boldsymbol{\psi}\left(\boldsymbol{u}^{\prime}\right)}+\boldsymbol{\phi}_{\overline{\boldsymbol{u}}}\left(\boldsymbol{u}^{\prime}\right)+\boldsymbol{\phi}_{\boldsymbol{u}^{\prime}}(\overline{\boldsymbol{u}}) \quad \text { in } \Omega, \\
& \boldsymbol{u}^{\prime}=\mathbf{0} \quad \text { on } \Gamma_{\text {cyl }},
\end{aligned}
$$

with arbitrary initial condition, defining $\left(\boldsymbol{u}^{\prime}, p^{\prime}\right)$ as a solution to the unsteady Navier-Stokes equations forced by (minus) the Reynolds stresses and two additional terms describing, respectively, the 
advection $\left(\boldsymbol{\phi}_{\bar{u}}\left(\boldsymbol{u}^{\prime}\right)=-\nabla \boldsymbol{u}^{\prime} \cdot \overline{\boldsymbol{u}}\right)$ and the production $\left(\boldsymbol{\phi}_{\boldsymbol{u}^{\prime}}(\overline{\boldsymbol{u}})=-\nabla \overline{\boldsymbol{u}} \cdot \boldsymbol{u}^{\prime}\right)$ of fluctuations by the mean flow. If we return to the adjoint systems (A1) and (A2), we have now

$$
\left(\left(\nabla \overline{u^{\dagger}} \cdot \boldsymbol{u}^{\prime}-\nabla \boldsymbol{u}^{\prime \mathrm{T}} \cdot \overline{\boldsymbol{u}^{\dagger}} \mid \delta \boldsymbol{u}^{\prime}\right)\right)=(\overline{\boldsymbol{u}^{\dagger}} \mid \underbrace{-\overline{\nabla \delta \boldsymbol{u}^{\prime} \cdot \boldsymbol{u}^{\prime}}-\overline{\nabla \boldsymbol{u}^{\prime} \cdot \delta \boldsymbol{u}^{\prime}}}_{\overline{\delta \psi\left(\boldsymbol{u}^{\prime}\right)}}),
$$

by integration by parts, meaning the source terms labeled (a) in (A1) and (A2) relate physically to the modification of the Reynolds stresses induced by the control force. In the same vein, we have

$$
\left(\left(\nabla \boldsymbol{u}^{\dagger \prime} \cdot \bar{u} \mid \delta u^{\prime}\right)\right)-\left(\overline{\nabla u^{\prime T} \cdot u^{\dagger \prime}} \mid \overline{\delta u}\right)=\left((u^{\dagger \prime} \mid \underbrace{-\nabla \delta u^{\prime} \cdot \bar{u}-\nabla u^{\prime} \cdot \overline{\delta u}}_{\delta \phi_{\bar{u}}\left(u^{\prime}\right)})\right),
$$

meaning terms labeled (b1)-(b2) relate to the modification of the advection mechanism. Finally, we have

$$
\left(\overline{\nabla u^{\dagger \prime} \cdot u^{\prime}} \mid \overline{\delta u}\right)-\left(\left(\nabla \bar{u}^{\mathrm{T}} \cdot u^{\dagger \prime} \mid \delta u^{\prime}\right)\right)=\left((u^{\dagger \prime} \mid \underbrace{-\nabla \overline{\delta u} \cdot u^{\prime}-\nabla \bar{u} \cdot \delta u^{\prime}}_{\delta \phi_{u^{\prime}}(\bar{u})})\right),
$$

meaning terms labeled (c1)-(c2) relate to the modification of the production mechanism.

The solutions to Eqs. (A1) and (A2) can be expanded into the sum of homogeneous and particular solutions (note we refer by "homogeneous" to the right-hand side of the adjoint equations of motions, not to the boundary condition at the cylinder surface that keeps being non-homogeneous). On the mean side, the homogeneous solution is precisely that $\left(\overline{\boldsymbol{u}_{0}^{\dagger}}, \overline{p_{0}^{\dagger}}\right)$ computed in our simplified framework. On the fluctuating side, the homogeneous solution is inferred from (A2) to be trivially zero, which reflects the fact that the mean drag does not depend explicitly on the fluctuation to the mean flow perturbation. We are thus left with

$$
\left(\overline{\boldsymbol{u}^{\dagger}}, \overline{p^{\dagger}}\right)=\left(\overline{\boldsymbol{u}_{0}^{\dagger}}, \overline{p_{0}^{\dagger}}\right)+\left(\overline{\boldsymbol{u}_{\psi}^{\dagger}}, \overline{p_{\psi}^{\dagger}}\right) \quad \text { and } \quad\left(\boldsymbol{u}^{\dagger \prime}, p^{\dagger \prime}\right)=\left(\boldsymbol{u}_{\psi}^{\dagger \prime}, p_{\psi}^{\dagger \prime}\right) \text {, }
$$

where the subscripts " $\psi$ " indicate the particular solutions. This allows recasting the mean drag variation into

$$
\overline{\delta D}=\left(\left(\boldsymbol{u}^{\dagger} \mid \boldsymbol{\delta} \boldsymbol{f}\right)\right)=\underbrace{\left(\overline{\boldsymbol{u}_{0}^{\dagger}} \mid \overline{\boldsymbol{\delta} \boldsymbol{f}}\right)}_{\overline{\delta D_{0}}}+\underbrace{\left(\overline{\boldsymbol{u}_{\psi}^{\dagger}} \mid \overline{\boldsymbol{\delta} \boldsymbol{f}}\right)+\left(\left(\boldsymbol{u}_{\psi}^{\dagger \prime} \mid \boldsymbol{\delta} \boldsymbol{f}^{\prime}\right)\right)}_{\overline{\delta D_{\psi}}},
$$

this new form being best suited to discuss the various physical mechanisms at stakes and the degree of approximation they achieve:

(i) The first term $\left(\overline{\boldsymbol{u}_{0}^{\dagger}} \mid \overline{\boldsymbol{\delta} \boldsymbol{f}}\right)$ in (A8) is that taken into account in our simplified analysis. It stems from the mean flow modification induced explicitly by the mean part of the control force, in the exact same way as the base flow is modified by a steady force in Sec. III.

(ii) The second term $\left(\overline{\boldsymbol{u}_{\psi}^{\dagger}} \mid \overline{\boldsymbol{\delta} \boldsymbol{f}}\right)$ in (A8) is due to the fact that the above mean flow disturbance modifies the advection and production of fluctuations. Therefore a fluctuating perturbation is induced, that in turn feeds back on the mean flow perturbation through the Reynolds stresses.

(iii) The third term $\left(\left(\boldsymbol{u}_{\psi}^{\dagger \prime} \mid \boldsymbol{\delta} \boldsymbol{f}^{\prime}\right)\right)$ is related to the fact that the fluctuating part of the force induces another fluctuating perturbation feeding back on the mean flow perturbation through the Reynolds stresses.

The terms discussed in points (ii) and (iii) are neglected in our simplified analysis. Their sum denoted by $\overline{\delta D_{\psi}}$ physically represents the correction from the approximated drag variation $\overline{\delta D_{0}}$ to the exact drag variation $\overline{\delta D}$ through the nonlinear coupling of the mean and fluctuating perturbation motions. This correction can be expressed as a function of the approximated sensitivity $\left(\overline{\boldsymbol{u}_{0}^{\dagger}}, \overline{p_{0}^{\dagger}}\right)$ according to

$$
\overline{\delta D_{\psi}}=\left(\overline{\boldsymbol{u}_{0}^{\dagger}} \mid \overline{\delta \psi\left(\boldsymbol{u}^{\prime}\right)}\right)
$$


since any arbitrary force can be used in the variational technique underlying the derivation of the adjoint problem (43), so we can choose in particular the modification of the Reynolds stresses induced by the control force itself. Of course, Eq. (A9), is not practically usable since knowledge of $\overline{\delta \boldsymbol{\psi}\left(\boldsymbol{u}^{\prime}\right)}$ requires to perform systematically DNS of the controlled flow, which is precisely what the adjoint method is meant to avoid.

\section{APPENDIX B: DERIVATION OF THE SENSITIVITY FUNCTIONS IN LAMINAR STEADY FLOW REGIME}

This appendix is devoted to the derivation of the various sensitivities used in Sec. III.

\section{Sensitivity of the steady asymptotic drag}

The quantity of interest is the steady asymptotic drag defined as

$$
D=2 \oint_{\Gamma_{\text {cyl }}}\left\{\boldsymbol{\sigma}\left(p_{b}, \boldsymbol{u}_{b}\right) \cdot \boldsymbol{n}\right\} \cdot \boldsymbol{e}_{\boldsymbol{x}} \mathrm{d} l
$$

where we denote by $\left(\boldsymbol{u}_{b}, p_{b}\right)$ the base solution to the forced, steady Navier-Stokes equations

$$
\begin{aligned}
\nabla \cdot \boldsymbol{u}_{b}=0, \quad \nabla \boldsymbol{u}_{b} \cdot \boldsymbol{u}_{b}-\nabla \cdot \boldsymbol{\sigma}\left(p_{b}, \boldsymbol{u}_{b}\right)=\boldsymbol{f} & \text { in } \Omega . \\
\boldsymbol{u}_{b}=\mathbf{0} & \text { on } \Gamma_{\text {cyl }},
\end{aligned}
$$

to be solved numerically with open flow boundary conditions on $\partial \Omega$ consisting of a uniform freestream $\boldsymbol{u}_{b}=\boldsymbol{e}_{\boldsymbol{x}}$ at the inflow, symmetric conditions $\partial_{y} u_{b}=v_{b}=0$ at the transverse boundaries and a stress-free condition $\boldsymbol{\sigma}\left(p_{b}, \boldsymbol{u}_{b}\right) \cdot \boldsymbol{n}=\mathbf{0}$ at the outflow. In this formulation, the natural cylinder flow considered in Sec. III is the solution to Eq. (B2) for $\boldsymbol{f}=\mathbf{0}$. By definition of the sensitivity function $\nabla_{f} D$ relative to the spatial $L_{2}$ inner product on $\Omega$, any small modification in the control force modifies drag by $\delta D$ according to

$$
\delta D=2 \oint_{\Gamma_{\mathrm{cyl}}}\left\{\boldsymbol{\sigma}\left(\delta p_{b}, \delta \boldsymbol{u}_{b}\right) \cdot \boldsymbol{n}\right\} \cdot \boldsymbol{e}_{\boldsymbol{x}} \mathrm{d} l=\int_{\Omega} \nabla_{\boldsymbol{f}} D \cdot \boldsymbol{\delta} \boldsymbol{f} \mathrm{d} x \mathrm{~d} y,
$$

where $\left(\delta \boldsymbol{u}_{b}, \delta p_{b}\right)$ is the linear perturbation to the base solution induced by the control force, such that

$$
\begin{aligned}
\nabla \cdot \delta \boldsymbol{u}_{b}=\mathbf{0}, \quad \nabla \delta \boldsymbol{u}_{b} \cdot \boldsymbol{u}_{b}+\nabla \boldsymbol{u}_{b} \cdot \boldsymbol{\delta} \boldsymbol{u}_{b}-\nabla \cdot \boldsymbol{\sigma}\left(\delta p_{b}, \boldsymbol{\delta} \boldsymbol{u}_{b}\right)=\boldsymbol{\delta} \boldsymbol{f} & \text { in } \Omega, \\
\boldsymbol{\delta} \boldsymbol{u}_{b}=\mathbf{0} & \text { on } \Gamma_{\text {cyl }},
\end{aligned}
$$

together with boundary conditions $\delta \boldsymbol{u}_{b}=\mathbf{0}$ at the inlet, $\partial_{y} \delta u_{b}=\delta v_{b}=0$ at the transverse boundaries and $\boldsymbol{\sigma}\left(\delta p_{b}, \boldsymbol{\delta} \boldsymbol{u}_{b}\right) \cdot \boldsymbol{n}=\mathbf{0}$ at the outflow.

In the present Lagrangian formalism, the control force $f$ is the control variable, the solution $\left(\boldsymbol{u}_{b}, p_{b}\right)$ is the state variable, and Eq. (B2) is the state equation, i.e., the constraint to be satisfied. We introduce Lagrange multipliers $\left(\boldsymbol{u}_{b}^{\dagger}, p_{b}^{\dagger}\right)$ referred to as the adjoint solution (also known as co-state variable), and define the functional

$$
\begin{aligned}
\mathcal{J}\left(\boldsymbol{u}_{b}, p_{b}, \boldsymbol{u}_{b}^{\dagger}, p_{b}^{\dagger}, \boldsymbol{f}\right) & =D \\
& -\int_{\Omega} p_{b}^{\dagger}\left\{\nabla \cdot \boldsymbol{u}_{b}\right\} \mathrm{d} x \mathrm{~d} y \\
& -\int_{\Omega} \boldsymbol{u}_{b}^{\dagger} \cdot\left\{\nabla \boldsymbol{u}_{b} \cdot \boldsymbol{u}_{b}-\nabla \cdot \boldsymbol{\sigma}\left(p_{b}, \boldsymbol{u}_{b}\right)-\boldsymbol{f}\right\} \mathrm{d} x \mathrm{~d} y,
\end{aligned}
$$

whose gradient with respect to any variable $s$ is

$$
\frac{\partial \mathcal{J}}{\partial s} \delta s=\lim _{\epsilon \rightarrow 0} \frac{\mathcal{J}((s+\epsilon \delta s)-\mathcal{J}(s)}{\epsilon} .
$$


We thus have

$$
\delta D=\frac{\partial \mathcal{J}}{\partial\left(\boldsymbol{u}_{b}, p_{b}\right)} \boldsymbol{\delta}\left(\boldsymbol{u}_{b}, p_{b}\right)+\frac{\partial \mathcal{J}}{\partial \boldsymbol{f}} \boldsymbol{\delta} \boldsymbol{f},
$$

since the gradient of the functional with respect to the adjoint variable is zero as long as the state equation is satisfied. The gradient with respect to the base solution $\left(\boldsymbol{u}_{b}, p_{b}\right)$ is

$$
\begin{aligned}
\frac{\partial \mathcal{J}}{\partial\left(\boldsymbol{u}_{b}, p_{b}\right)} \boldsymbol{\delta}\left(\boldsymbol{u}_{b}, p_{b}\right)= & 2 \oint_{\Gamma_{\text {cyl }}}\left\{\boldsymbol{\sigma}\left(\delta p_{b}, \delta \boldsymbol{u}_{b}\right) \cdot \boldsymbol{n}\right\} \cdot \boldsymbol{e}_{\boldsymbol{x}} \mathrm{d} l \\
& -\int_{\Omega} p_{b}^{\dagger}\left\{\nabla \cdot \delta \boldsymbol{u}_{b}\right\} \mathrm{d} x \mathrm{~d} y \\
& -\int_{\Omega} \boldsymbol{u}_{b}^{\dagger} \cdot\left\{\nabla \boldsymbol{\delta} \boldsymbol{u}_{b} \cdot \boldsymbol{u}_{b}+\nabla \boldsymbol{u}_{b} \cdot \boldsymbol{\delta} \boldsymbol{u}_{b}-\nabla \cdot \boldsymbol{\sigma}\left(\delta p_{b}, \boldsymbol{\delta} \boldsymbol{u}_{b}\right)\right\} .
\end{aligned}
$$

Integrating by parts and using the divergence theorem, we obtain

$$
\begin{aligned}
\frac{\partial \mathcal{J}}{\partial\left(\boldsymbol{u}_{b}, p_{b}\right)} \boldsymbol{\delta}\left(\boldsymbol{u}_{b}, p_{b}\right)= \\
\left.\quad \oint_{\Gamma_{\mathrm{cyl}}}\left(\left\{\boldsymbol{\sigma}\left(\delta p_{b}, \delta \boldsymbol{u}_{b}\right) \cdot \boldsymbol{n}\right\} \cdot\left\{2 \boldsymbol{e}_{x}-\boldsymbol{u}_{b}^{\dagger}\right\}+\left\{\boldsymbol{\sigma}\left(-p_{b}^{\dagger}, \boldsymbol{u}_{b}^{\dagger}\right) \cdot \boldsymbol{n}+\left(\boldsymbol{u}_{b} \cdot \boldsymbol{n}\right) \boldsymbol{u}_{b}^{\dagger}\right\} \cdot \delta \boldsymbol{u}_{b}\right)\right) \mathrm{d} l \\
+\oint_{\partial \Omega}\left(-\left\{\boldsymbol{\sigma}\left(\delta p_{b}, \boldsymbol{\delta} \boldsymbol{u}_{b}\right) \cdot \boldsymbol{n}\right\} \cdot \boldsymbol{u}_{b}^{\dagger}+\left\{\boldsymbol{\sigma}\left(-p_{b}^{\dagger}, \boldsymbol{u}_{b}^{\dagger}\right) \cdot \boldsymbol{n}+\left(\boldsymbol{u}_{b} \cdot \boldsymbol{n}\right) \boldsymbol{u}_{b}^{\dagger}\right\} \cdot \boldsymbol{\delta} \boldsymbol{u}_{b}\right) \mathrm{d} l \\
+\int_{\Omega}\left\{\nabla \cdot \boldsymbol{u}_{b}^{\dagger}\right\} \delta p_{b} \mathrm{~d} x \mathrm{~d} y \\
-\int_{\Omega}\left\{-\nabla \boldsymbol{u}_{b}^{\dagger} \cdot \boldsymbol{u}_{b}+\nabla \boldsymbol{u}_{b}^{\mathrm{T}} \cdot \boldsymbol{u}_{b}^{\dagger}-\nabla \cdot \boldsymbol{\sigma}\left(-p_{b}^{\dagger}, \boldsymbol{u}_{b}^{\dagger}\right)\right\} \cdot \boldsymbol{\delta} \boldsymbol{u}_{b} \mathrm{~d} x \mathrm{~d} y .
\end{aligned}
$$

Canceling the surface term on $\Omega$ and the boundary terms on $\Gamma_{\text {cyl }} \times \partial \Omega$ defines $\left(\boldsymbol{u}_{b}^{\dagger}, p_{b}^{\dagger}\right)$ as the solution to the linear equations

$$
\begin{array}{rrl}
\nabla \cdot \boldsymbol{u}_{b}^{\dagger}=\mathbf{0}, & -\nabla \boldsymbol{u}_{b}^{\dagger} \cdot \boldsymbol{u}_{b}+\nabla \boldsymbol{u}_{b}^{\mathrm{T}} \cdot \boldsymbol{u}_{b}^{\dagger}-\nabla \cdot \boldsymbol{\sigma}\left(-p_{b}^{\dagger}, \boldsymbol{u}_{b}^{\dagger}\right)=\mathbf{0} & \text { in } \Omega, \\
\boldsymbol{u}_{b}^{\dagger}=2 \boldsymbol{e}_{\boldsymbol{x}} & \text { on } \Gamma_{\mathrm{cyl}},
\end{array}
$$

together with adjoint boundary conditions $\boldsymbol{u}_{b}^{\dagger}=\mathbf{0}$ at the inflow, $\partial_{y} u_{b}^{\dagger}=v_{b}^{\dagger}=0$ at the transverse boundaries and $\boldsymbol{\sigma}\left(-p_{b}^{\dagger}, \boldsymbol{u}_{b}^{\dagger}\right)+\left(\boldsymbol{u}_{b} \cdot \boldsymbol{n}\right) \boldsymbol{u}_{b}^{\dagger}=\mathbf{0}$ at the outflow. In return, Eq. (B7) reduces to

$$
\delta D=\frac{\partial \mathcal{J}}{\partial \boldsymbol{f}} \boldsymbol{\delta} \boldsymbol{f}=\int_{\Omega} \boldsymbol{u}_{b}^{\dagger} \cdot \boldsymbol{\delta} \boldsymbol{f} \mathrm{d} x \mathrm{~d} y,
$$

and we deduce comparing (B3)-(B11) that the sensitivity function is simply

$$
\nabla_{f} D=u^{\dagger}
$$

\section{Validation}

In this section, we consider a model force localized at some position $\left(x_{\mathrm{c}}, y_{\mathrm{c}}\right)$, pointing in the stream-wise $x$ direction with amplitude $\alpha$ assumed small positive, thus reading

$$
\boldsymbol{\delta} \boldsymbol{f}_{\alpha}(x, y)=\alpha \delta\left(x-x_{\mathrm{c}}, y-y_{\mathrm{c}}\right) \boldsymbol{e}_{\boldsymbol{x}},
$$

where $\delta(x, y)$ stands for 2D Dirac delta function. For validation purposes of our sensitivity calculations, we compare the theoretical value of the steady asymptotic drag variation divided by the 
TABLE I. Variation of steady asymptotic drag divided by the amplitude parameter for the $+x$ pointing model force defined by (B13) - $R e=40$. Theoretical values computed from Eq. (B14) are reported in Column 3. Columns 4 and 5 provide, respectively, theoretical values and finite difference values estimated from base flow computation of the controlled solutions, both computed smoothing out numerically the model force into Gaussian (B15).

\begin{tabular}{|c|c|c|c|c|}
\hline$x_{\mathrm{c}}$ & $y_{\mathrm{c}}$ & $u_{b}^{\dagger}\left(x_{\mathrm{c}}, y_{\mathrm{c}}\right)$ & $\left(\boldsymbol{u}_{b}^{\dagger} \mid \delta \hat{f}_{\alpha}\right) / \alpha$ & $\left(D_{\alpha}-D\right) / \alpha$ \\
\hline-1.5 & 0 & $2.32 \times 10^{0}$ & $2.32 \times 10^{0}$ & $2.33 \times 10^{0}$ \\
\hline 0 & 0.65 & $7.10 \times 10^{-1}$ & $7.10 \times 10^{-1}$ & $7.11 \times 10^{-1}$ \\
\hline 0 & 0.85 & $-2.89 \times 10^{-1}$ & $-2.89 \times 10^{-1}$ & $-2.84 \times 10^{-1}$ \\
\hline 1.5 & 0 & $1.24 \times 10^{0}$ & $1.24 \times 10^{0}$ & $1.24 \times 10^{0}$ \\
\hline 2.5 & 0 & $8.96 \times 10^{-1}$ & $8.96 \times 10^{-1}$ & $8.96 \times 10^{-1}$ \\
\hline 4 & 0 & $5.68 \times 10^{-1}$ & $5.68 \times 10^{-1}$ & $5.68 \times 10^{-1}$ \\
\hline
\end{tabular}

amplitude parameter computed in the frame of the current sensitivity analysis as

$$
\frac{\delta D}{\alpha}\left(\alpha, x_{\mathrm{c}}, y_{\mathrm{c}}\right)=\frac{1}{\alpha}\left(\boldsymbol{u}_{b}^{\dagger} \mid \boldsymbol{\delta} \boldsymbol{f}_{\alpha}\right)=u_{b}^{\dagger}\left(x_{\mathrm{c}}, y_{\mathrm{c}}\right),
$$

to the finite difference approximation $\left(D_{\alpha}-D\right) / \alpha$, where we denote by $D_{\alpha}$ the steady asymptotic drag of the controlled base solution computed with the Newton-Raphson method using the exact same method and boundary conditions as described in Sec. III B, but smoothing out numerically the body force into a Gaussian

$$
\boldsymbol{\delta} \hat{\boldsymbol{f}}_{\alpha}(x, y)=\frac{\alpha}{2 \pi \chi^{2}} \exp \left(\frac{\left(x-x_{\mathrm{c}}\right)^{2}+\left(y-y_{\mathrm{c}}\right)^{2}}{2 \chi^{2}}\right) \boldsymbol{e}_{\boldsymbol{x}},
$$

of standard deviation $\chi=6.25 \times 10^{-3}$. We have checked the norm of the Gaussian to depart from its theoretical value $\alpha$ by less than $1 \%$, and the results to change by less than $0.5 \%$ when either halving or doubling the value of $\chi$, which provides good evidence of relevance. We report in Table I numerical values obtained at representative locations $\left(x_{\mathrm{c}}, y_{\mathrm{c}}\right)$ distributed around the cylinder, either upstream of the cylinder, in the shear layers, or in the recirculation region. The drag sensitivities obtained using the Dirac function are exactly the same as their numerical counterpart computed from the exact inner product between the drag sensitivity and the Gaussian function, the change being only in the fourth decimal. Moreover, we obtain remarkable agreement with the finite difference values obtained for a small amplitude $\alpha=10^{-3}$, which validates the analysis as well as the accuracy of the computed sensitivity.

\section{Sensitivity of the steady asymptotic lift}

If we now consider the steady asymptotic lift

$$
L=2 \oint_{\Gamma_{\mathrm{cyl}}}\left\{\boldsymbol{\sigma}\left(p_{b}, \boldsymbol{u}_{b}\right) \cdot \boldsymbol{n}\right\} \cdot \boldsymbol{e}_{\boldsymbol{y}} \mathrm{d} l
$$

the sensitivity $\nabla_{f} L$ such that

$$
\delta L=2 \oint_{\Gamma_{\mathrm{cy} 1}}\left\{\boldsymbol{\sigma}\left(\delta p_{b}, \delta \boldsymbol{u}_{b}\right) \cdot \boldsymbol{n}\right\} \cdot \boldsymbol{e}_{\boldsymbol{y}} \mathrm{d} l=\int_{\Omega} \nabla_{f} L \cdot \boldsymbol{\delta} \boldsymbol{f} \mathrm{d} x \mathrm{~d} y,
$$

is obtained applying the exact same procedure to the functional

$$
\begin{aligned}
\mathcal{J}\left(\boldsymbol{u}_{b}, p_{b}, \boldsymbol{u}_{b}^{\dagger}, p_{b}^{\dagger}, \boldsymbol{f}\right) & =L \\
& -\int_{\Omega} p_{b}^{\dagger}\left\{\nabla \cdot \boldsymbol{u}_{b}\right\} \mathrm{d} x \mathrm{~d} y \\
& -\int_{\Omega} \boldsymbol{u}_{b}^{\dagger} \cdot\left\{\nabla \boldsymbol{u}_{b} \cdot \boldsymbol{u}_{b}-\nabla \cdot \boldsymbol{\sigma}\left(p_{b}, \boldsymbol{u}_{b}\right)-\boldsymbol{f}\right\} \mathrm{d} x \mathrm{~d} y .
\end{aligned}
$$


This amounts to substituting $\boldsymbol{e}_{\boldsymbol{y}}$ for $\boldsymbol{e}_{\boldsymbol{x}}$ in all preceding relations, which yields the same adjoint equations as (B10) together with boundary condition $\boldsymbol{u}_{b}^{\dagger}=2 \boldsymbol{e}_{\boldsymbol{y}}$ at the cylinder surface.

\section{APPENDIX C: DERIVATION OF THE SENSITIVITY FUNCTIONS IN LAMINAR UNSTEADY FLOW REGIME}

This third appendix is devoted to the derivation of the unsteady sensitivities used in Sec. IV.

\section{Sensitivity of the mean drag}

The quantity of interest is now the mean drag defined as

$$
\bar{D}=\frac{2}{\tau} \int_{0}^{\tau} \oint_{\Gamma_{\mathrm{cyl}}}\{\boldsymbol{\sigma}(p, \boldsymbol{u}) \cdot \boldsymbol{n}\} \cdot \boldsymbol{e}_{\boldsymbol{x}} \mathrm{d} l \mathrm{~d} t
$$

where we denote by $(\boldsymbol{u}, p)$ the solution to the forced, unsteady Navier-Stokes equations

$$
\begin{array}{rrl}
\nabla \cdot \boldsymbol{u}=0, & \partial_{t} \boldsymbol{u}+\nabla \boldsymbol{u} \cdot \boldsymbol{u}-\nabla \cdot \boldsymbol{\sigma}(p, \boldsymbol{u})=\boldsymbol{f} & \text { in } \Omega, \\
\boldsymbol{u}=\mathbf{0} & \text { on } \Gamma_{\mathrm{cyl}},
\end{array}
$$

to be solved numerically with open flow boundary conditions on $\partial \Omega$ consisting of a uniform freestream $\boldsymbol{u}=\boldsymbol{e}_{\boldsymbol{x}}$ at the inflow, symmetric conditions $\partial_{y} u=v=0$ at the transverse boundaries and an advective condition at the outflow. In this formulation, the natural cylinder flow considered in Sec. IV is the solution to Eq. (C2) for $\boldsymbol{f}=\mathbf{0}$. By definition of the sensitivity function $\nabla_{f} \bar{D}$ relative to the tensorized $L_{2}$ inner product on the time-space domain $(0, \tau) \times \Omega$, any small modification in the control force modifies drag by $\overline{\delta D}$ according to

$$
\overline{\delta D}=\frac{2}{\tau} \int_{0}^{\tau} \oint_{\Gamma_{\mathrm{cyl}}}\{\boldsymbol{\sigma}(\delta p, \delta \boldsymbol{u}) \cdot \boldsymbol{n}\} \cdot \boldsymbol{e}_{\boldsymbol{x}} \mathrm{d} l \mathrm{~d} t=\frac{1}{\tau} \int_{0}^{\tau} \int_{\Omega} \nabla_{f} \bar{D} \cdot \boldsymbol{\delta} \boldsymbol{f} \mathrm{d} x \mathrm{~d} y \mathrm{~d} t
$$

where $(\boldsymbol{\delta} \boldsymbol{u}, \delta p)$ is the instantaneous linear perturbation to the cylinder flow such that

$$
\begin{array}{rll}
\nabla \cdot \delta \boldsymbol{u}=\mathbf{0}, & \partial_{t} \delta \boldsymbol{u}+\nabla \delta \boldsymbol{u} \cdot \boldsymbol{u}+\nabla \boldsymbol{u} \cdot \delta \boldsymbol{u}-\nabla \cdot \boldsymbol{\sigma}(\delta p, \delta \boldsymbol{u})=\boldsymbol{\delta} \boldsymbol{f} & \text { in } \Omega, \\
\delta \boldsymbol{u}=\mathbf{0} & \text { on } \Gamma_{\mathrm{cyl}}, \\
\delta \boldsymbol{u}=\mathbf{0} & \text { at } t=0,
\end{array}
$$

together with boundary conditions $\delta \boldsymbol{u}=\mathbf{0}$ at the inlet, $\partial_{y} \delta u=\delta v=0$ at the transverse boundaries and a stress-free condition $\boldsymbol{\sigma}(\delta p, \delta \boldsymbol{u}) \cdot \boldsymbol{n}=\mathbf{0}$ at the outflow.

We define the new functional

$$
\begin{aligned}
\mathcal{J}\left(\boldsymbol{u}, p, \boldsymbol{u}^{\dagger}, p^{\dagger}, \boldsymbol{f}\right) & =\bar{D} \\
& -\frac{1}{\tau} \int_{0}^{\tau} \int_{\Omega} p^{\dagger}\{\nabla \cdot \boldsymbol{u}\} \mathrm{d} x \mathrm{~d} y \mathrm{dt} \\
& -\frac{1}{\tau} \int_{0}^{\tau} \int_{\Omega} \boldsymbol{u}^{\dagger} \cdot\left\{\partial_{t} \boldsymbol{u}+\nabla \boldsymbol{u} \cdot \boldsymbol{u}-\nabla \cdot \boldsymbol{\sigma}(p, \boldsymbol{u})-\boldsymbol{f}\right\} \mathrm{d} x \mathrm{~d} y \mathrm{dt},
\end{aligned}
$$

and repeat the above procedure starting from

$$
\overline{\delta D}=\frac{\partial \mathcal{J}}{\partial(\boldsymbol{u}, p)} \boldsymbol{\delta}(\boldsymbol{u}, p)+\frac{\partial \mathcal{J}}{\partial \boldsymbol{f}} \boldsymbol{\delta} \boldsymbol{f} .
$$


Integrating by parts the gradient with respect to the solution $(\boldsymbol{u}, p)$ and using the divergence theorem, we obtain

$$
\begin{aligned}
\frac{\partial \mathcal{J}}{\partial(\boldsymbol{u}, p)} \boldsymbol{\delta}(\boldsymbol{u}, p)= \\
\quad \frac{1}{\tau} \int_{0}^{\tau} \oint_{\Gamma_{\mathrm{cyl}}}\left(\{\boldsymbol{\sigma}(\delta p, \delta \boldsymbol{u}) \cdot \boldsymbol{n}\} \cdot\left\{2 \boldsymbol{e}_{\boldsymbol{x}}-\boldsymbol{u}^{\dagger}\right\}+\left\{\boldsymbol{\sigma}\left(-p^{\dagger}, \boldsymbol{u}^{\dagger}\right) \cdot \boldsymbol{n}+(\boldsymbol{u} \cdot \boldsymbol{n}) \boldsymbol{u}^{\dagger}\right\} \cdot \boldsymbol{\delta} \boldsymbol{u}\right) \mathrm{d} l \mathrm{dt} \\
\quad+\frac{1}{\tau} \int_{0}^{\tau} \oint_{\partial \Omega}\left(-\{\boldsymbol{\sigma}(\delta p, \delta \boldsymbol{u}) \cdot \boldsymbol{n}\} \cdot \boldsymbol{u}^{\dagger}+\left\{\boldsymbol{\sigma}\left(-p^{\dagger}, \boldsymbol{u}^{\dagger}\right) \cdot \boldsymbol{n}+(\boldsymbol{u} \cdot \boldsymbol{n}) \boldsymbol{u}^{\dagger}\right\} \cdot \boldsymbol{\delta} \boldsymbol{u}\right) \mathrm{d} l \mathrm{dt} \\
\quad+\frac{1}{\tau} \int_{0}^{\tau} \int_{\Omega}\left\{\nabla \cdot \boldsymbol{u}^{\dagger}\right\} \delta p \mathrm{~d} x \mathrm{~d} y \mathrm{dt} \\
\quad-\frac{1}{\tau} \int_{0}^{\tau} \int_{\Omega}\left\{-\partial_{t} \boldsymbol{u}^{\dagger}-\nabla \boldsymbol{u}^{\dagger} \cdot \boldsymbol{u}+\nabla \boldsymbol{u}^{\mathrm{T}} \cdot \boldsymbol{u}^{\dagger}-\nabla \cdot \boldsymbol{\sigma}\left(-p^{\dagger}, \boldsymbol{u}^{\dagger}\right)\right\} \cdot \boldsymbol{\delta} \boldsymbol{u} \mathrm{d} x \mathrm{~d} y \mathrm{dt} \\
\quad-\frac{1}{\tau} \int_{\Omega}\left[\boldsymbol{u}^{\dagger} \cdot \boldsymbol{\delta} \boldsymbol{u}\right]_{0}^{\tau} \mathrm{d} x \mathrm{~d} y .
\end{aligned}
$$

Canceling the surface term on $\Omega$ and the boundary terms on $\Gamma_{\text {cyl }} \times \partial \Omega$ defines $\left(\boldsymbol{u}^{\dagger}, p^{\dagger}\right)$ as the solution to the linear equations

$$
\begin{array}{rll}
\nabla \cdot \boldsymbol{u}^{\dagger}=\mathbf{0}, & -\partial_{t} \boldsymbol{u}^{\dagger}-\nabla \boldsymbol{u}^{\dagger} \cdot \boldsymbol{u}+\nabla \boldsymbol{u}^{\mathrm{T}} \cdot \boldsymbol{u}^{\dagger}-\nabla \cdot \sigma\left(-p^{\dagger}, \boldsymbol{u}^{\dagger}\right)=\mathbf{0} & \text { in } \Omega, \\
\boldsymbol{u}^{\dagger}=2 \boldsymbol{e}_{\boldsymbol{x}} & \text { on } \Gamma_{\mathrm{cyl}},
\end{array}
$$

with same boundary conditions on $\partial \Omega$ as defined in Appendix B 1, namely, $\boldsymbol{u}^{\dagger}=\mathbf{0}$ at the inflow, $\partial_{y} u^{\dagger}=v^{\dagger}=0$ at the transverse boundaries and $\boldsymbol{\sigma}\left(-p^{\dagger}, \boldsymbol{u}^{\dagger}\right)+(\boldsymbol{u} \cdot \boldsymbol{n}) \boldsymbol{u}^{\dagger}=\mathbf{0}$ at the outflow. The only terms to survive in (C7) are those

$$
-\frac{1}{\tau} \int_{\Omega}\left[\boldsymbol{u}^{\dagger} \cdot \boldsymbol{\delta} \boldsymbol{u}\right]_{0}^{\tau} \mathrm{d} x \mathrm{~d} y=-\frac{1}{\tau}\left\{\boldsymbol{u}^{\dagger}(\tau) \cdot \boldsymbol{\delta} \boldsymbol{u}(\tau)-\boldsymbol{u}^{\dagger}(0) \cdot \boldsymbol{\delta} \boldsymbol{u}(0)\right\},
$$

stemming from the time derivatives, whose cancellation requires imposing $\boldsymbol{u}^{\dagger}(\tau)=\mathbf{0}$ since $\boldsymbol{\delta} \boldsymbol{u}(0)=\mathbf{0}$. In return, Eq. (C6) reduces to

$$
\overline{\delta D}=\frac{\partial \mathcal{J}}{\partial \boldsymbol{f}} \boldsymbol{\delta} \boldsymbol{f}=\frac{1}{\tau} \int_{0}^{\tau} \int_{\Omega} \boldsymbol{u}^{\dagger} \cdot \boldsymbol{\delta} \boldsymbol{f} \mathrm{d} x \mathrm{~d} y \mathrm{~d} t
$$

and we deduce comparing (C3)-(C10) that the sensitivity function is simply

$$
\nabla_{f} \bar{D}=\boldsymbol{u}^{\dagger}
$$

\section{Validation}

We return to the steady, $+x$ pointing model force (B13), and compare the theoretical value of the mean drag variation divided by the amplitude parameter computed in the frame of the current sensitivity analysis as

$$
\frac{\overline{\delta D}}{\alpha}\left(\alpha, x_{\mathrm{c}}, y_{\mathrm{c}}\right)=\frac{1}{\alpha}\left(\left(\boldsymbol{u}^{\dagger} \mid \boldsymbol{\delta} \boldsymbol{f}_{\alpha}\right)\right)=\frac{1}{\alpha}\left(\overline{\boldsymbol{u}^{\dagger}} \mid \boldsymbol{\delta} \boldsymbol{f}_{\alpha}\right)=\overline{u^{\dagger}}\left(x_{\mathrm{c}}, y_{\mathrm{c}}\right),
$$

to the finite difference approximation $\left(\bar{D}_{\alpha}-\bar{D}\right) / \alpha$, where we denote by $\bar{D}_{\alpha}$ the mean drag of the controlled solution, computed by DNS smoothing out the body force into Gaussian (B15) with same standard deviation $\chi=6.25 \times 10^{-3}$. We report in Table II numerical values obtained at representative locations $\left(x_{\mathrm{c}}, y_{\mathrm{c}}\right)$ distributed around the cylinder, either upstream of the cylinder, in the shear layers, or in the recirculation region. The drag sensitivities obtained using the Dirac function are almost identical to their numerical counterpart computed from the exact inner product between the drag sensitivity and the Gaussian function, the change being only in the third decimal. Moreover, we 
TABLE II. Variation of mean drag divided by the amplitude parameter for the $+x$ pointing model force defined by (B13) $R e=100$. Theoretical values computed from Eq. (C12) are reported in Column 3. Columns 4 and 5 provide, respectively, theoretical values and finite difference values estimated by DNS of the controlled solutions, both computed smoothing out numerically the model force into Gaussian (B15).

\begin{tabular}{|c|c|c|c|c|}
\hline$x_{\mathrm{c}}$ & $y_{\mathrm{c}}$ & $\overline{u^{\dagger}}\left(x_{\mathrm{c}}, y_{\mathrm{c}}\right)$ & $\left(\left(\boldsymbol{u}^{\dagger} \mid \delta \hat{f}_{\alpha}\right) / \alpha\right.$ & $\left(\bar{D}_{\alpha}-\bar{D}\right) / \alpha$ \\
\hline-1.5 & 0 & $3.27 \times 10^{0}$ & $3.27 \times 10^{0}$ & $3.33 \times 10^{0}$ \\
\hline 0.1 & 0.725 & $-2.09 \times 10^{0}$ & $-2.08 \times 10^{0}$ & $-2.07 \times 10^{0}$ \\
\hline 0.65 & 0 & $1.59 \times 10^{0}$ & $1.59 \times 10^{0}$ & $1.58 \times 10^{0}$ \\
\hline 1.5 & 0 & $-1.38 \times 10^{0}$ & $-1.38 \times 10^{0}$ & $-1.38 \times 10^{0}$ \\
\hline 2.6 & 0 & $2.54 \times 10^{-1}$ & $2.57 \times 10^{-1}$ & $2.62 \times 10^{-1}$ \\
\hline
\end{tabular}

obtain a very good agreement between the adjoint-based predictions and the finite difference values obtained for a small amplitude $\alpha=10^{-3}$ (a small discrepancy by $2 \%$ is noticed at the most upstream position where the mesh is coarser owing to numerical constraints), which validates the analysis as well as the accuracy of the computed sensitivity.

\section{Sensitivity of the steady mean lift}

If we now consider the mean lift

$$
\bar{L}=\frac{2}{\tau} \int_{0}^{\tau} \oint_{\Gamma_{\mathrm{cyl}}}\{\boldsymbol{\sigma}(p, \boldsymbol{u}) \cdot \boldsymbol{n}\} \cdot \boldsymbol{e}_{\boldsymbol{y}} \mathrm{d} l \mathrm{~d} t
$$

the sensitivity $\nabla_{f} \bar{L}$ such that

$$
\overline{\delta L}=\frac{2}{\tau} \int_{0}^{\tau} \oint_{\Gamma_{\mathrm{cyl}}}\{\boldsymbol{\sigma}(\delta p, \delta \boldsymbol{u}) \cdot \boldsymbol{n}\} \cdot \boldsymbol{e}_{\boldsymbol{y}} \mathrm{d} l \mathrm{~d} t=\frac{1}{\tau} \int_{0}^{\tau} \int_{\Omega} \nabla_{f} \bar{L} \cdot \boldsymbol{\delta} \boldsymbol{f} \mathrm{d} x \mathrm{~d} y \mathrm{~d} t
$$

is obtained applying the exact same procedure to the functional

$$
\begin{aligned}
\mathcal{J}\left(\boldsymbol{u}, p, \boldsymbol{u}^{\dagger}, p^{\dagger}, \boldsymbol{f}\right) & =\bar{L} \\
& -\frac{1}{\tau} \int_{0}^{\tau} \int_{\Omega} p^{\dagger}\{\nabla \cdot \boldsymbol{u}\} \mathrm{d} x \mathrm{~d} y \mathrm{dt} \\
& -\frac{1}{\tau} \int_{0}^{\tau} \int_{\Omega} \boldsymbol{u}^{\dagger} \cdot\left\{\partial_{t} \boldsymbol{u}+\nabla \boldsymbol{u} \cdot \boldsymbol{u}-\nabla \cdot \boldsymbol{\sigma}(p, \boldsymbol{u})-\boldsymbol{f}\right\} \mathrm{d} x \mathrm{~d} y \mathrm{dt} .
\end{aligned}
$$

This again amounts to substituting $\boldsymbol{e}_{\boldsymbol{y}}$ for $\boldsymbol{e}_{\boldsymbol{x}}$ in all preceding relations, which yields the same adjoint equations as (C8) together with boundary condition $\boldsymbol{u}^{\dagger}=2 \boldsymbol{e}_{\boldsymbol{y}}$ at the cylinder surface.

\section{Sensitivity of the rms drag and lift}

The sensitivity of the rms drag is obtained similarly provided the variation $\delta D_{\text {rms }}$ is recast into

$$
\delta D_{\mathrm{rms}}=\frac{1}{\tau} \int_{0}^{\tau} \frac{D^{\prime}}{D_{\mathrm{rms}}} \delta D \mathrm{~d} t=\frac{2}{\tau} \int_{0}^{\tau} \oint_{\Gamma_{\mathrm{cyl}}} \frac{D^{\prime}}{D_{\mathrm{rms}}}\{\boldsymbol{\sigma}(\delta p, \delta \boldsymbol{u}) \cdot \boldsymbol{n}\} \cdot \boldsymbol{e}_{\boldsymbol{x}} \mathrm{d} l \mathrm{~d} t,
$$

whereupon the exact same procedure is applied to the functional

$$
\begin{aligned}
\mathcal{J}\left(\boldsymbol{u}, p, \boldsymbol{u}^{\dagger}, p^{\dagger}, \boldsymbol{f}\right) & =D_{\mathrm{rms}} \\
& -\int_{\Omega} p^{\dagger}\{\nabla \cdot \boldsymbol{u}\} \mathrm{d} x \mathrm{~d} y \\
& -\int_{\Omega} \boldsymbol{u}^{\dagger} \cdot\left\{\partial_{t} \boldsymbol{u}+\nabla \boldsymbol{u} \cdot \boldsymbol{u}-\nabla \cdot \boldsymbol{\sigma}(p, \boldsymbol{u})-\boldsymbol{f}\right\} \mathrm{d} x \mathrm{~d} y .
\end{aligned}
$$


Integrating by parts the gradient with respect to the solution $(\boldsymbol{u}, p)$ and using the divergence theorem, we obtain

$$
\begin{aligned}
\frac{\partial \mathcal{J}}{\partial(\boldsymbol{u}, p)} \boldsymbol{\delta}(\boldsymbol{u}, p)= \\
\quad \frac{1}{\tau} \int_{0}^{\tau} \oint_{\Gamma_{\mathrm{cyl}}}\left(\{\boldsymbol{\sigma}(\delta p, \delta \boldsymbol{u}) \cdot \boldsymbol{n}\} \cdot\left\{2 \frac{D^{\prime}}{D_{\mathrm{rms}}} \boldsymbol{e}_{\boldsymbol{x}}-\boldsymbol{u}^{\dagger}\right\}+\left\{\boldsymbol{\sigma}\left(-p^{\dagger}, \boldsymbol{u}^{\dagger}\right) \cdot \boldsymbol{n}+(\boldsymbol{u} \cdot \boldsymbol{n}) \boldsymbol{u}^{\dagger}\right\} \cdot \boldsymbol{\delta} \boldsymbol{u}\right) \mathrm{d} l \mathrm{dt} \\
+\frac{1}{\tau} \int_{0}^{\tau} \oint_{\partial \Omega}\left(-\{\boldsymbol{\sigma}(\delta p, \delta \boldsymbol{u}) \cdot \boldsymbol{n}\} \cdot \boldsymbol{u}^{\dagger}+\left\{\boldsymbol{\sigma}\left(-p^{\dagger}, \boldsymbol{u}^{\dagger}\right) \cdot \boldsymbol{n}+(\boldsymbol{u} \cdot \boldsymbol{n}) \boldsymbol{u}^{\dagger}\right\} \cdot \boldsymbol{\delta} \boldsymbol{u}\right) \mathrm{d} l \mathrm{dt} \\
+\frac{1}{\tau} \int_{0}^{\tau} \int_{\Omega}\left\{\nabla \cdot \boldsymbol{u}^{\dagger}\right\} \delta p \mathrm{~d} x \mathrm{~d} y \mathrm{dt} \\
\quad-\frac{1}{\tau} \int_{0}^{\tau} \int_{\Omega}\left\{-\partial_{t} \boldsymbol{u}^{\dagger}-\nabla \boldsymbol{u}^{\dagger} \cdot \boldsymbol{u}+\nabla \boldsymbol{u}^{\mathrm{T}} \cdot \boldsymbol{u}^{\dagger}-\nabla \cdot \boldsymbol{\sigma}\left(-p^{\dagger}, \boldsymbol{u}^{\dagger}\right)\right\} \cdot \delta \boldsymbol{u} \mathrm{d} x \mathrm{~d} y \mathrm{dt} \\
\quad-\frac{1}{\tau} \int_{\Omega}\left[\boldsymbol{u}^{\dagger} \cdot \boldsymbol{\delta} \boldsymbol{u}\right]_{0}^{\tau} \mathrm{d} x \mathrm{~d} y
\end{aligned}
$$

which yields the same adjoint equations of motion as (C1) together with boundary condition $\boldsymbol{u}^{\dagger}=2 D^{\prime} / D_{\mathrm{rms}}$ at the cylinder surface. The sensitivity of the rms lift is obtained similarly substituting $\boldsymbol{e}_{\boldsymbol{y}}$ for $\boldsymbol{e}_{\boldsymbol{x}}$ in all preceding relations, which yields the same adjoint equations of motion together with boundary condition $\boldsymbol{u}^{\dagger}=2 L^{\prime} / L_{\mathrm{rms}}$ at the cylinder surface.

${ }^{1}$ P. J. Strykowski and K. R. Sreenivasan, "On the formation and suppression of vortex shedding at low Reynolds numbers," J. Fluid Mech. 218, 71 (1990).

${ }^{2}$ H. B. Kim and K. S. Chang, "Numerical study on vortex shedding from a circular cylinder influenced by a nearby control wire," Comput. Fluid Dyn. J. 4, 151 (1995).

${ }^{3}$ S. Mittal and A. Raghuvanshi, "Control of vortex shedding behind circular cylinder for flows at low Reynolds number," Intl. J. Numer. Meth. Fluids 35, 421 (2001).

${ }^{4}$ M. Morzynski, K. Afanasiev, and F. Thiele, "Solution of the eigenvalue problems resulting from global non-parallel flow stability analysis," Comput. Meth. Appl. Mech. Eng. 169, 161 (1999).

${ }^{5}$ C. Dalton, Y. Xu, and J. C. Owen, "The suppression of lift on a circular cylinder due to vortex shedding at moderate Reynolds numbers,” J. Fluids Struct. 15, 617 (2001).

${ }^{6}$ I. Yildirim, C. C. M. Rindt, and A. A. Steenhoven, "Vortex dynamics in a wire-disturbed cylinder wake," Phys. Fluids 22 , 094101 (2010).

${ }^{7}$ H. Sakamoto, K. Tan, and H. Haniu, "An optimum suppression of fluid forces by controlling a shear layer separated from a square prism,” ASME Trans. J. Fluids Eng. 113, 183 (1991).

${ }^{8}$ H. Sakamoto and H. Haniu, "Optimum suppression of fluid forces acting on circular cylinder," ASME Trans. J. Fluids Eng. 116, 221 (1994).

${ }^{9}$ T. Igarashi, "Drag reduction of a square prism by flow control using a small rod," J. Wind Eng. Ind. Aerodyn. 69, 141 (1997).

${ }^{10}$ V. Parezanović and O. Cadot, "The impact of a local perturbation on global properties of a turbulent wake," Phys. Fluids 21, 071701 (2009).

${ }^{11}$ V. Parezanović and O. Cadot, "Experimental sensitivity analysis of the global properties of a 2D turbulent wake," J. Fluid Mech. 693, 115 (2012).

${ }^{12}$ O. Cadot, B. Thiria, and J.-F. Beaudoin, "Passive drag control of a turbulent wake by local disturbances," in Unsteady Separated Flows and their Control, edited by M. Braza and K. Hourigan (Springer, 2009), p. 529.

${ }^{13}$ D. C. Hill, "A theoretical approach for analyzing the restabilization of wakes," NASA Technical Report No. 103858, 1992.

${ }^{14}$ O. Marquet, D. Sipp, and L. Jacquin, "Sensitivity analysis and passive control of cylinder flow," J. Fluid Mech. 615, 221 (2008).

${ }^{15}$ C. P. Jackson, "A finite-element study of the onset of vortex shedding in flow past variously shaped bodies," J. Fluid Mech. 182, 23 (1987).

${ }^{16}$ A. Zebib, "Stability of a viscous flow past a circular cylinder," J. Eng. Math. 21, 155 (1987).

${ }^{17}$ P. Luchini, F. Giannetti, and J. Pralits, "Structural sensitivity of linear and nonlinear global modes," AIAA Paper No. 2008-4227, 2008.

${ }^{18}$ O. Marquet, D. Sipp, L. Jacquin, and J.-M. Chomaz, "Multiple scale and sensitivity analysis for the passive control of the cylinder flow," AIAA Paper No. 2008-4228, 2008.

${ }^{19}$ J. Pralits, L. Brandt, and F. Giannetti "Instability and sensitivity of the flow around a rotating circular cylinder," J. Fluid Mech. 650, 513 (2010).

${ }^{20}$ P. Meliga and J.-M. Chomaz, "Global modes in a confined impinging jet: application to heat transfer and control," Theor. Comput. Fluid Dyn. 25, 179 (2010). 
${ }^{21}$ F. Giannetti and P. Luchini, "Structural sensitivity of the first instability of the cylinder wake," J. Fluid Mech. 581, 167 (2007).

${ }^{22}$ P. Meliga, D. Sipp, and J.-M. Chomaz, "Open-loop control of compressible afterbody flows using adjoint methods," Phys. Fluids 22, 054109 (2010).

${ }^{23}$ D. Sipp, O. Marquet, P. Meliga, and A. Barbagallo, "Dynamics and control of global instabilities in open-flows: a linearized approach," App. Mech. Rev. 63, 030801 (2010).

${ }^{24}$ O. Tammisola, "Oscillatory sensitivity patterns for global modes in wakes," J. Fluid Mech. 701, 251 (2012).

${ }^{25}$ P. Luchini and A. Bottaro, "Adjoint equations in stability analysis," Annu. Rev. Fluid Mech. 46, 493 (2014).

${ }^{26}$ P. Meliga, G. Pujals, and E. Serre, "Sensitivity of 2D turbulent flow past a D-shaped cylinder using global stability," Phys. Fluids 24, 061701 (2012).

${ }^{27}$ C. Mettot, D. Sipp, and H. Bezard, "Quasi-laminar stability and sensitivity analyses for turbulent flows: Prediction of low-frequency unsteadiness and passive control," Phys. Fluids 26, 045112 (2014).

${ }^{28}$ A. Jameson, L. Martinelli, and N. A. Pierce, "Optimum aerodynamic design using the Navier-Stokes equations," Theor. Comput. Fluid Dyn. 10, 213 (1998).

${ }^{29}$ A. Jameson, "Optimum aerodynamic design using CFD and control theory," AIAA Paper No. 95-1729-807, 1995.

${ }^{30}$ J. Hoffman, "Computation of mean drag for bluff body problems using adaptive DNS/LES," SIAM J. Sci. Comput. 27, 184 (2005).

${ }^{31}$ J. Hoffman, "Adaptive simulation of the subcritical flow past a sphere," J. Fluid Mech. 568, 77 (2006).

${ }^{32}$ Q. Wang and J.-H. Gao, "The drag-adjoint field of a circular cylinder wake at Reynolds numbers 20, 100 and 500," J. Fluid Mech. 730, 145 (2013).

${ }^{33}$ K. M. Kelkar and S. V. Patankar, "Numerical prediction of vortex shedding behind a square cylinder," Int. J. Numer. Meth. Fluids 14, 327 (1992).

${ }^{34}$ A. Sohankar, C. Norberg, and L. Davidson, "Numerical simulation of unsteady low-Reynolds number flow around rectangular cylinders at incidence," J. Wind Eng. Ind. Aerodyn. 69-71, 189 (1997).

${ }^{35}$ A. Sohankar, C. Norberg, and L. Davidson, "Low-Reynolds-number flow around a square cylinder at incidence: study of blockage, onset of vortex shedding and outlet boundary condition,” Int. J. Numer. Meth. Fluids 26, 39 (1998).

${ }^{36}$ M. D. Gunzburger, "Sensitivities, adjoints and flow optimization,” Int. J. Numer. Methods Fluids 31, 53 (1999)

${ }^{37}$ F. Hecht, "New development in freefem++," J. Numer. Math. 20, 251 (2012).

${ }^{38}$ T. A. Davis and I. S. Duff, "An unsymmetric-pattern multifrontal method for sparse lu factorization," SIAM J. Matrix Anal. Appl. 18, 140 (1997).

${ }^{39}$ T. A. Davis, "A column pre-ordering strategy for the unsymmetric-pattern multifrontal method," ACM Trans. Math. Softw. 30, 165 (2004).

${ }^{40}$ M. Breuer, J. Bernsdorf, T. Zeiser, and F. Durst, "Accurate computations of the laminar flow past a square cylinder based on two different methods: lattice-Boltzmann and finite-volume," Int. J. Heat Fluid Flow 21, 186 (2000).

${ }^{41}$ D. J. Tritton, "Experiments on the flow past a circular cylinder at low Reynolds numbers," J. Fluid Mech. 6, 547 (1959).

${ }^{42}$ B. Fornberg, "A numerical study of steady viscous flow past a circular cylinder," J. Fluid Mech. 98, 819 (1980).

${ }^{43}$ P. Luchini, F. Giannetti, and J. Pralits, "Structural sensitivity of the finite-amplitude vortex shedding behind a circular cylinder," in Unsteady Separated Flows and their Control, edited by M. Braza and K. Hourigan (Springer, 2009), p. 151.

${ }^{44}$ J. Robichaux, S. Balachandar, and S. P. Vanka, "Three-dimensional Floquet instability of the wake of square cylinder," Phys. Fluids 11, 560 (1999).

${ }^{45}$ R. D. Blevins, Flow-Induced Vibration, 2nd ed. (Van Nostrand Reinhold, New York, 1990).

${ }^{46}$ D. W. Barkley, "Linear analysis of the cylinder wake mean flow," Europhys. Lett. 75, 750 (2006).

${ }^{47}$ A. Maurel, V. Pagneux, and J. E. Wesfreid, "Mean-flow correction as non-linear saturation mechanism," Europhys. Lett. 32, 217 (1995).

${ }^{48}$ B. J. A. Zielinska, S. Goujon-Durand, J. Dusek, and J. E. Wesfreid, "Strongly nonlinear effect in unstable wakes," Phys. Rev. Lett. 79, 3893 (1997)

${ }^{49}$ B. R. Noack, K. Afanasiev, M. Morzynski, G. Tadmor, and F. Thiele, "A hierarchy of low-dimensional models for the transient and post-transient cylinder wake," J. Fluid Mech. 497, 335 (2003).

${ }^{50}$ B. Thiria and J. E. Wesfreid, "Stability properties of forced wakes," J. Fluid Mech. 579, 137 (2007).

${ }^{51}$ D. A. Hammond and L. G. Redekopp, "Global dynamics of symmetric and asymmetric wakes," J. Fluid Mech. 331, 231 (1997).

${ }^{52}$ D. Sipp and A. Lebedev, "Global stability of base and mean flows: a general approach and its applications to cylinder and open cavity flows," J. Fluid Mech. 593, 333 (2007).

${ }^{53}$ M. Khor, J. Sheridan, M. C. Thompson, and K. Hourigan, "Global frequency selection in the observed time-mean wakes of circular cylinders," J. Fluid Mech. 601, 425 (2008).

${ }^{54}$ P. Meliga, D. Sipp, and J.-M. Chomaz, "Elephant modes and low frequency unsteadiness in a high Reynolds number, transonic afterbody wake," Phys. Fluids 21, 054105 (2009).

${ }^{55}$ J. S. Leontini, M. C. Thompson, and K. Hourigan, "A numerical study of global frequency selection in the time-mean wake of a circular cylinder," J. Fluid Mech. 645, 435 (2010).

${ }^{56}$ Y. Hwang and C. Cossu, "Amplification of coherent streaks in the turbulent Couette flow: an input-output analysis at low Reynolds number," J. Fluid Mech. 643, 333 (2010).

${ }^{57}$ M. Marquillie, U. Ehrenstein, and J.-P. Laval, "Instability of streaks in wall turbulence with adverse pressure gradient," J. Fluid Mech. 681, 205 (2011).

${ }^{58}$ D. J. Lea, A. Myles, and T. W. N. Haines, "Sensitivity analysis of the climate of a chaotic system," Tellus A 52, 523 (2000).

${ }^{59} \mathrm{~A}$. Köhl and J. Willebrand, "An adjoint method for the assimilation of statistical characteristics into eddy-resolving ocean models," Tellus A 54, 406 (2002).

${ }^{60}$ T. J. Barth, "On the role of error and uncertainty in the numerical simulation of complex fluid flow," in Lecture Notes in Computational Science and Engineering (Springer, 2007), Vol. 56, p. 29. 
${ }^{61}$ M. Nazarov and J. Hoffman, "On the stability of the dual problem for high Reynolds number flow past a circular cylinder in two dimensions," SIAM J. Sci. Comput. 34, A1905 (2012).

${ }^{62}$ W. Rodi, "Comparison of LES and RANS calculations of the flow around bluff bodies," J. Wind Eng. Ind. Aerodyn. 69-71, 55 (1997).

${ }^{63}$ G. Iaccarino, A. Ooi, P. A. Durbin, and M. Behnia, "Reynolds averaged simulation of unsteady separated flow," Int. J. Heat Fluid Flow 24, 147 (2003)

${ }^{64}$ P. R. Spalart and S. R. Allmaras, "A one-equation turbulence model for aerodynamic flows," Recherche Aérospatiale 1, 5 (1994); also AIAA Paper No. 92-0439, 1992.

${ }^{65}$ Source code and official documentation available at http://www.openfoam.org.

${ }^{66}$ R. I. Issa, "Solution of the implicitly discretized fluid flow equation by operator splitting," J. Comput. Phys. 62, 40 (1986).

${ }^{67}$ See supplementary material at http://dx.doi.org/10.1063/1.4896941 for provision of the theoretical framework and detailed equations solved in Ref. 26.

${ }^{68}$ Y. Bao, D. Zhou, Q. Wu, and X.-Q. Chen, "Numerical prediction of aerodynamic characteristics of prismatic cylinder by finite element method with Spalart-Allmaras turbulence model," J. Comput. Struct. 89, 325 (2011).

${ }^{69}$ W. Rodi, J. H. Ferziger, M. Breuer, and M. Pourquie, “Status of large-eddy simulation: results of a workshop,” J. Fluids Eng. 248, 55 (1997).

${ }^{70}$ D. A. Lyn, S. Einav, W. Rodi, and J. H. Park, "A laser-Doppler velocimetry study of ensemble averaged characteristics of the turbulent near wake of a square cylinder," J. Fluid Mech. 304, 285 (1995).

${ }^{71}$ B. E. Lee "The effect of turbulence on the surface pressure field of a square prism," J. Fluid Mech. 69, 263 (1975).

${ }^{72}$ M. Minguez, C. Brun, R. Pasquetti, and E. Serre, "Experimental and high-order LES analysis of the flow in near-wall region of a square cylinder," Int. J. Heat Fluid Flow 32, 558 (2011).

${ }^{73}$ A. Sohankar, L. Davidson, and C. Norberg, "Large eddy simulation of flow past a square cylinder: comparison of different subgrid scale models," ASME Trans. J. Fluids Eng. 122, 39 (2000).

${ }^{74}$ R. D. Henderson, "Details of the drag curve near the onset of vortex shedding," Phys. Fluids 7, 2102 (1995).

${ }^{75}$ Note systems (A1) and (A2) could be derived equivalently applying the variational technique used in Sec. IV, the mean and fluctuating adjoint fields being then viewed as respective Lagrange multipliers for the steady and unsteady problems obtained linearizing Eqs. (41)-(A3) - in doing so, one must yet pay attention to the fact that the unsteady adjoint system is then defined up to a zero-mean function. 